\title{
Convergence of Adaptive Finite Element Methods*
}

\author{
Pedro Morin ${ }^{\dagger}$ \\ Ricardo H. Nochetto \\ Kunibert G. Siebert ${ }^{\S}$
}

\begin{abstract}
Adaptive finite element methods (FEMs) have been widely used in applications for over 20 years now. In practice, they converge starting from coarse grids, although no mathematical theory has been able to prove this assertion. Ensuring an error reduction rate based on a posteriori error estimators, together with a reduction rate of data oscillation (information missed by the underlying averaging process), we construct a simple and efficient adaptive FEM for elliptic partial differential equations. We prove that this algorithm converges with linear rate without any preliminary mesh adaptation nor explicit knowledge of constants. Any prescribed error tolerance is thus achieved in a finite number of steps. A number of numerical experiments in two and three dimensions yield quasi-optimal meshes along with a competitive performance. Extensions to higher order elements and applications to saddle point problems are discussed as well.
\end{abstract}

Key words. a posteriori error estimators, data oscillation, adaptive mesh refinement, convergence, Stokes problem, Uzawa iterations

AMS subject classifications. $65 \mathrm{~N} 12,65 \mathrm{~N} 15,65 \mathrm{~N} 30,65 \mathrm{~N} 50,65 \mathrm{Y} 20$

PII. S0036144502409093

I. Introduction and Main Result. Adaptive procedures for the numerical solution of partial differential equations (PDEs) started in the late 1970s and are now standard tools in science and engineering. We refer to [21] for references on adaptivity for elliptic PDEs, and restrict the list of papers to those strictly related to our work. Adaptive finite element methods (FEMs) are indeed a meaningful approach for handling multiscale phenomena and making realistic computations feasible, especially in three dimensions.

A posteriori error estimators are an essential ingredient of adaptivity. They are computable quantities depending on the computed solution(s) and data that provide

*Published electronically October 30, 2002. This paper originally appeared in SIAM Journal on Numerical Analysis, Volume 38, Number 2, 2000, pages 466-488. Most of this work was developed while the first and third authors were visiting the University of Maryland.

http://www.siam.org/journals/sirev/44-4/40909.html

†Instituto de Matemática Aplicada del Litoral, UNL - CONICET, Güemes 3450, 3000 Santa Fe, Argentina (pmorin@math.unl.edu.ar). This author was partially supported by Programa FOMECUNL, CONICET of Argentina, and NSF DMS-9971450 and INT-9910086.

${ }^{\ddagger}$ Department of Mathematics and Institute for Physical Science and Technology, University of Maryland, College Park, MD 20742 (rhn@math.umd.edu). This author was partially supported by NSF DMS-9971450 and INT-9910086.

$\S$ Institut für Angewandte Mathematik, Hermann-Herder-Str. 10, 79104 Freiburg, Germany (kgs@mathematik.uni-freiburg.de). This author was partially supported by DAAD-NSF grant "Projektbezogene Förderung des Wissenschaftleraustauschs in den Natur-, Ingenieur- und den Sozialwissenschaften mit der NSF." 
information about the quality of approximation and may thus be used to make judicious mesh modifications. The ultimate purpose is to construct a sequence of meshes that will eventually equidistribute the approximation errors and, as a consequence, the computational effort. To this end, the a posteriori error estimators are split into element indicators which are then employed to make local mesh modifications by refinement (and sometimes coarsening). This naturally leads to loops of the form

$$
\text { Solve } \rightarrow \text { Estimate } \rightarrow \text { Refine. }
$$

Experience strongly suggests that, starting from a coarse mesh, such an iteration converges within any prescribed error tolerance in a finite number of steps. Except for the rather complete description of the one-dimensional situation by Babuška and Vogelius [2], convergence of (1.1) in the multidimensional case is largely an open issue. The fundamental paper [8] of Dörfler for the Poisson equation shows a linear error reduction rate for the energy norm towards a preassigned tolerance $\epsilon$ in finite steps, provided that

(a) the initial mesh is sufficiently refined to resolve data within a tolerance $\mu \epsilon \ll \epsilon$ (mesh fineness);

(b) the sum of the local error indicators of elements marked for refinement amounts to a fixed proportion of the global error estimator (marking strategy).

We also refer to [9] and [11] for related work. Our results are based on, and in fact improve upon, this idea of Dörfler. They were first published in [16] and are reviewed and slightly improved here as well as extended to saddle point problems.

To be more specific, let $\Omega$ be a polygonal (polyhedral) bounded domain of $\mathbb{R}^{d}$, for $d=2,3$, and let $u$ be the solution to the following problem:

$$
\begin{aligned}
-\operatorname{div}(A \nabla u)=f & \text { in } \Omega, \\
u=0 & \text { on } \partial \Omega,
\end{aligned}
$$

where $f \in L^{2}(\Omega)$ and $A$ is a piecewise constant positive definite symmetric matrix. Let $\mathcal{T}_{H}$ be a conforming triangulation of $\Omega$, with piecewise constant mesh-size $H$, and let $u_{H}$ denote the piecewise linear finite element solution over $\mathcal{T}_{H}$ assuming exact integration. This is an ideal situation which helps isolate essential difficulties from quadrature issues; the latter deserve a separate investigation. The following quantity, hereafter called data oscillation, will play a fundamental role:

$$
\operatorname{osc}\left(f, \mathcal{T}_{H}\right):=\left\|H\left(f-f_{H}\right)\right\|_{\Omega}:=\left(\sum_{T \in \mathcal{T}_{H}}\left\|H\left(f-f_{T}\right)\right\|_{T}^{2}\right)^{1 / 2} ;
$$

from now on $f_{H}$ stands for a piecewise constant approximation of $f$ over $\mathcal{T}_{H}$ with element value $f_{T}$ equal to the mean value of $f$ over $T \in \mathcal{T}_{H}$. Such $\operatorname{osc}\left(f, \mathcal{T}_{H}\right)$ measures intrinsic information missing in the averaging process associated with finite elements, which fail to detect fine structures of $f$. We stress that (1.3) is unrelated to quadrature and quantifies data oscillation with the least amount of information per element, namely one degree of freedom associated with $f_{T}$. Its relation with the infamous saturation assumption is explored in [10].

In contrast to [8], which imposes the constraint $\|H f\|_{\Omega} \leq \mu \epsilon$ on the interior residual as a notion of mesh fineness in (a), we claim that a fixed reduction rate of $\operatorname{osc}\left(f, \mathcal{T}_{H}\right)$ in the adaptive procedure is what really matters; this crucial property is 
derived in section 3 . We observe that $\operatorname{osc}\left(f, \mathcal{T}_{H}\right)$ is generically of higher order than the interior residual $\|H f\|_{\Omega}$. The original condition $\|H f\|_{\Omega} \leq \mu \epsilon$ of [8] may easily lead to an initial overrefinement (see section 5.2). As illustrated in Example 3.8, there might be pathological instances when data oscillation and interior residual are comparable and the concept of data oscillation reduction becomes relevant. We propose simple but essential changes to [8] in order to avoid a restriction of mesh fineness. This results in a practical procedure, Algorithm C of section 3, whose formulation needs no tolerance $\epsilon$. In addition to marking elements according to (b) in each step, Algorithm C starts from any coarse mesh and ensures one interior node for each marked element together with a linear data oscillation decay. The interior node guarantees suitable error decrease when data oscillation is relatively small. Its need is justified in Examples 3.6 and 3.7 , and a proof of the fundamental error reduction property is given in section 4 . Algorithm C yields the following convergence result, proved in section 3.

Main Result. Let $\left\{u_{k}\right\}_{k \in \mathbb{N}_{0}}$ be a sequence of piecewise linear finite element solutions in nested finite element spaces $\left\{\mathbb{V}_{k}\right\}_{k \in \mathbb{N}_{0}}$ produced by Algorithm C. There exist positive constants $C_{0}$ and $\beta<1$, depending only on given data and the initial grid, such that

$$
\left\|u-u_{k}\right\|_{\Omega} \leq C_{0} \beta^{k}
$$

where the energy norm is given by $\|v\|_{\Omega}=\left(\int_{\Omega} \nabla v \cdot A \nabla v\right)^{1 / 2}$. The initial coarse mesh need not be adjusted to resolve data to any tolerance, and no explicit constants are needed for Algorithm $C$ to work.

A few comments and comparisons are now in order.

- Any prescribed error tolerance $\epsilon$ may be met in finite steps, as in [8], but without any special tuning of the initial mesh in terms of $\epsilon$; this issue is important and its practical implications are fully examined in section 5.2.

- Depending on the flatness of $u$, the mesh-size $H$ may not necessarily tend to zero, which makes this a nonstandard finite element asymptotic statement.

- Even though no stability constants are required for Algorithm C, nor for convergence, the constant in the upper bound of the residual-type a posteriori error estimate is needed to stop the iterations; this is customary in adaptivity $[21]$.

- Inequality (1.4) does not imply that the error decays in every single step as in [8], and Example 3.8 shows that it may be constant for a number of steps due to unresolved data oscillation.

- The interior node created by Algorithm C guarantees suitable error decrease when data oscillation is small and is discussed in Examples 3.6 and 3.7. This theoretical requirement may be viewed as a looking-ahead strategy, and the two- and three-dimensional experiments of section 5 clearly indicate no additional computational expense.

- Both Algorithm C and the main result extend to higher order finite element discretizations (see section 6) and are instrumental for the application to the Stokes problem (see section 7 ).

In section 5 we present several illuminating numerical experiments computed within the finite element toolbox ALBERT [19, 20]. The first example is the crack problem, which exhibits a point singularity of the form $r^{1 / 2}$. The second example corresponds to intersecting interfaces (discontinuous coefficients) and a much more severe singularity of the form $r^{\gamma}$ with $\gamma$ arbitrarily close to 0 . In both cases $f$ is 
constant and thus $\operatorname{osc}\left(f, \mathcal{T}_{H}\right)=0$. We finally discuss a two- and three-dimensional example with variable $f$ and thereby test the effect of data oscillation. It turns out that this effect seems to play a minor role for smooth $f$ (relative to the underlying mesh). This provides a heuristic explanation for the success of most adaptive strategies which disregard the issue of data oscillation altogether. The experiments also reveal that the resulting meshes are quasi-optimal: the error decays proportionally to $N^{-1 / d}$, $N$ being the number of degrees of freedom. Our theory, however, does not explain this important property. Mesh optimality has been recently studied in [5] via wavelet algorithms which resort to the entire wavelet expansions of $u$ and $f$. These full expansions are not available in practice, though, and somehow encode the missing information of data oscillation.

This paper is organized as follows. In section 2 we recall the standard a posteriori residual-type error estimators which constitute the core of adaptivity [21]. In section 3 we present Algorithm C, justify its various steps, and prove the main result. In section 4 we derive the crucial error reduction property, thereby closing the gap. We present several two- and three-dimensional experiments in section 5 which fully document the competitive performance of our novel adaptive strategy. We briefly discuss how to extend these ideas to higher order finite elements in section 6 , and conclude in section 7 with an application to saddle point problems via the Uzawa algorithm with emphasis on the Stokes equation.

2. A Posteriori Error Estimators. We start this section with some useful notation. For an open set $G \subset \mathbb{R}^{d}$ we denote by $H^{1}(G)$ the usual Sobolev space of functions in $L^{2}(G)$ whose first derivatives are also in $L^{2}(G)$, endowed with the norm

$$
\|u\|_{H^{1}(G)}:=\left(\|u\|_{G}^{2}+\|\nabla u\|_{G}^{2}\right)^{1 / 2},
$$

where $\|\cdot\|_{G}$ stands for the $L^{2}(G)$-norm.

Since $A$ is piecewise constant, symmetric, and positive definite, the bilinear form $a_{G}(\cdot, \cdot)$ defined for any open set $G \subset \Omega$ by

$$
a_{G}(u, v):=\int_{G} A \nabla u \cdot \nabla v
$$

is bounded, and $a(\cdot, \cdot):=a_{\Omega}(\cdot, \cdot)$ is coercive on $H_{0}^{1}(\Omega)$; i.e., there exist constants $0<c_{a} \leq C_{a}<\infty$ such that

$$
\begin{gathered}
a_{G}(v, w) \leq C_{a}\|v\|_{H^{1}(G)}\|w\|_{H^{1}(G)} \quad \forall v, w \in H^{1}(G), \\
c_{a}\|v\|_{H^{1}(\Omega)}^{2} \leq a(v, v) \quad \forall v \in H_{0}^{1}(\Omega) .
\end{gathered}
$$

This implies, in particular, that the seminorm $\|\cdot\|_{G}$ defined by $\|v\|_{G}^{2}:=a_{G}(v, v)$ is equivalent to the $H_{0}^{1}(\Omega)$-norm when $G=\Omega$. We also define for any measurable set $G$

$$
\langle v, w\rangle_{G}:=\int_{G} v w
$$

In view of (2.1) and (2.2), problem (1.2) admits a unique weak solution $u$ for any $f \in L^{2}(\Omega)$; i.e.,

$$
u \in H_{0}^{1}(\Omega): \quad a(u, v)=\langle f, v\rangle_{\Omega} \quad \forall v \in H_{0}^{1}(\Omega) .
$$


Let $\mathcal{T}_{H}$ be a conforming regular triangulation of $\Omega$, with piecewise constant meshsize $H$, i.e., $\left.H\right|_{T}=\operatorname{diam}(T)$. Let $\mathbb{V}^{H}$ be the space of continuous piecewise linear functions over $\mathcal{T}_{H}$, and let $\mathbb{V}_{0}^{H}$ be the subspace of functions of $\mathbb{V}^{H}$ that vanish at the boundary. Let $u_{H}$ denote the solution of the discrete problem

$$
u_{H} \in \mathbb{V}_{0}^{H}: \quad a\left(u_{H}, \phi\right)=\langle f, \phi\rangle_{\Omega} \quad \forall \phi \in \mathbb{V}_{0}^{H} .
$$

The function $f_{H}$ will denote the piecewise constant function over $\mathcal{T}_{H}$ that, restricted to each element $T \in \mathcal{T}_{H}$, is equal to the mean value $f_{T}$ of $f$ on $T$.

We denote by $\mathcal{S}_{H}$ the set of sides of the triangulation $\mathcal{T}_{H}$ that do not belong to the boundary $\partial \Omega$ of the domain $\Omega$. For $S \in \mathcal{S}_{H}$, the domain $\omega_{S}$ is the union of the two elements in $\mathcal{T}_{H}$ sharing $S$, and $H_{S}$ denotes the diameter of $S$. We assume that all partitions $\mathcal{T}_{H}$ match the discontinuities of $A$; i.e., the jumps of $A$ are located on interelement boundaries $S \in \mathcal{S}_{H}$.

We now recall the residual-type a posteriori error estimators for (2.3) and (2.4) [1], [21]. Subtracting (2.4) from (2.3) and integrating by parts yields the following well-known relation between the error $u-u_{H}$ and the residuals:

$$
a\left(u-u_{H}, \phi\right)=\sum_{T \in \mathcal{T}_{H}}\left\langle f, \phi-\mathcal{I}_{H} \phi\right\rangle_{T}+\sum_{S \in \mathcal{S}_{H}}\left\langle J_{S}, \phi-\mathcal{I}_{H} \phi\right\rangle_{S} \quad \forall \phi \in H_{0}^{1}(\Omega) .
$$

Hereafter, $\mathcal{I}_{H}$ denotes the Clément interpolation operator, and $J_{S}=\llbracket A \nabla u_{H} \rrbracket_{S} \cdot \nu$ represents the jump of flux across side $S$ which is independent of the orientation of the unit normal $\nu$. Let $\eta_{S}$ be the (local) error indicator associated with side $S \in \mathcal{S}_{H}$,

$$
\eta_{S}^{2}:=\left\|H_{S}^{\frac{1}{2}} J_{S}\right\|_{S}^{2}+\|H f\|_{\omega_{S}}^{2},
$$

which consists of the jump and interior residuals, respectively. For any given subset $\tilde{\mathcal{S}}_{H} \subset \mathcal{S}_{H}$ we define

$$
\eta\left(u_{H}, \tilde{\mathcal{S}}_{H}\right)^{2}:=\sum_{S \in \tilde{\mathcal{S}}_{H}} \eta_{S}^{2}
$$

Upon taking $\phi=u-u_{H}$ in (2.5), and using interpolation theory, the following residualtype error estimator emerges [1], [21].

Lemma 2.1 (upper bound). There exists a constant $C_{1}$, depending only on the minimum angle of $\mathcal{T}_{H}, C_{a}$, and $c_{a}$, such that

$$
\left\|u-u_{H}\right\|_{\Omega}^{2} \leq C_{1} \eta\left(u_{H}, \mathcal{S}_{H}\right)^{2} .
$$

An early indication of the crucial role of oscillation is given by the (local) lower bound [1], [21]. The construction by Verfürth [21] will be important later.

Lemma 2.2 (lower bound). There exist constants $C_{2}, C_{3}$, depending only on the minimum angle of $\mathcal{T}_{H}, C_{a}$, and $c_{a}$, such that

$$
C_{2} \eta_{S}^{2}-C_{3}\left\|H\left(f-f_{H}\right)\right\|_{\omega_{S}}^{2} \leq\left\|u-u_{H}\right\|_{\omega_{S}}^{2} .
$$

Remark 2.3. Suppose that $\left\|H\left(f-f_{H}\right)\right\|_{\omega_{S}}$ is small with respect to $\eta_{S}$. In such a case, the significance of (2.8) is that the size of $\eta_{S}$ provides reliable information about the size of $\left\|u-u_{H}\right\|_{\omega_{S}}$. This explains why refining elements with large error indicators usually tends to equidistribute approximation errors, an ultimate goal of adaptivity.

Remark 2.4. It is worth realizing that $f_{H}$ in (2.8) could be any piecewise polynomial and not just the mean value of $f$ over the elements. This additional freedom in measuring data oscillation disappears in dealing with consecutive discrete solutions $u_{H}$ and $u_{h}$. This issue will be central to our discussions of section 4 . 
3. Algorithm C and Convergence. We start with a marking strategy for error reduction due to Dörfler [8]:

\section{Marking Strategy E}

Given a parameter $0<\theta<1$ :

1. Construct a subset $\hat{\mathcal{S}}_{H}$ of $\mathcal{S}_{H}$ such that

$$
\eta\left(u_{H}, \hat{\mathcal{S}}_{H}\right) \geq \theta \eta\left(u_{H}, \mathcal{S}_{H}\right) .
$$

2. Mark the subset $\hat{\mathcal{T}}_{H}$ of $\mathcal{T}_{H}$ of elements with one side in $\hat{\mathcal{S}}_{H}$.

This marking strategy ensures that we choose sufficiently many sides $S$ such that their contributions $\eta_{S}$ constitute a fixed proportion of the global error estimator $\eta\left(u_{H}, \mathcal{S}_{H}\right)$. Using Marking Strategy E, we have the following error reduction result, which is proved in section 4 .

THEOREM 3.1 (error reduction). Let $\mathcal{T}_{H}$ be a triangulation of $\Omega$, and let $\hat{\mathcal{T}}_{H}$ be defined in Marking Strategy E. Let $\mathcal{T}_{h}$ be a refinement of $\mathcal{T}_{H}$ satisfying $\mathbb{V}^{H} \subset \mathbb{V}^{h}$ and suppose that

each element of $\hat{\mathcal{T}}_{H}$, as well as each of its faces, contains a node of $\mathcal{T}_{h}$ in its interior.

Then there exists a constant $0<\alpha<1$, depending only on the minimum angle, $\theta$, $C_{a}$, and $c_{a}$, such that the solution $u_{h}$ on the mesh $\mathcal{T}_{h}$ satisfies

$$
\left\|u-u_{h}\right\|_{\Omega} \leq \alpha\left\|u-u_{H}\right\|_{\Omega}+\operatorname{osc}\left(f, \mathcal{T}_{H}\right) .
$$

Remark 3.2. The condition $\mathbb{V}^{H} \subset \mathbb{V}^{h}$ is crucial for error reduction. Using refinement by bisection directly leads to a sequence of nested spaces [14, 15]. Using the procedure of regular (red) refinement with green closure does not result in nested spaces due to removal of the green closure before a new refinement takes place [21]. In addition, creating an interior node for each element of $\hat{\mathcal{T}}_{H}$ by bisection produces, as a by-product, at least one interior node in each of the element sides (see section 5.1).

Remark 3.3. Theorem 3.1 establishes an error reduction provided the current error is much larger than data oscillation in the sense that

$$
\operatorname{osc}\left(f, \mathcal{T}_{H}\right) \leq \mu\left\|u-u_{H}\right\|_{\Omega}
$$

for some constant $\mu<1-\alpha$; in fact this implies

$$
\left\|u-u_{h}\right\|_{\Omega} \leq \beta\left\|u-u_{H}\right\|_{\Omega}, \quad \beta=\alpha+\mu<1 .
$$

In [8], Dörfler proves a similar result but assuming that

$$
\|H f\|_{\Omega} \leq \mu\left\|u-u_{H}\right\|_{\Omega}
$$

instead of (3.2). The notion of mesh fineness given by (3.2) is generically of higher order than (3.4), and thus easier to achieve in practice. This is the key idea in [16].

Remark 3.4. The requirement of having a node of the refined grid $\mathcal{T}_{h}$ inside each marked element of $\mathcal{T}_{H}$ is an essential difference to [8]. This requirement is responsible for the new measure of data approximation. Its implementation in two and three dimensions by bisection is discussed in section 5 . 

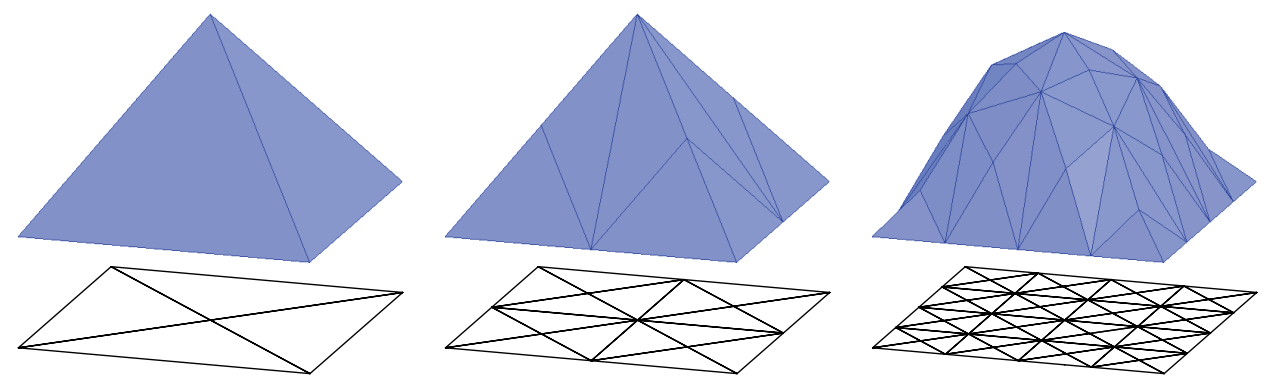

Fig. 3.I Example 3.6: Finite element solutions $u_{0}, u_{1}, u_{2}$ for three consecutive meshes $\mathcal{T}_{0}, \mathcal{T}_{1}, \mathcal{T}_{2}$ obtained with two bisection steps. The triangles of $\mathcal{T}_{1}$ do not have interior nodes, but those of $\mathcal{T}_{2}$ do, thereby yielding $u_{1}=u_{2}=\frac{1}{12} \phi_{1} \neq u_{3}$.

Remark 3.5. A repeated application of Marking Strategy E and the special refinement (3.1) of Theorem 3.1 would lead to an iterative process that reduces the error according to (1.4), provided (3.2) were valid for all iterations; this is the case of $f$ constant because $\operatorname{osc}\left(f, \mathcal{T}_{H}\right)=0$. Consequently, any error tolerance $\epsilon$ could be achieved in finite steps. In this vein, the main contribution of $[8]$ is to preadapt the initial mesh $\mathcal{T}_{0}$ according to $\left\|h_{0} f\right\|_{\Omega} \leq \mu \epsilon$ and show that (1.4) holds until $\left\|u-u_{h}\right\| \leq$ $\epsilon$. However, if this process were to be restarted with a stringent tolerance, neither (3.2) nor (3.4) may be satisfied; this happens, for instance, in section 7. Consequently, this strategy alone does not yield an asymptotically convergent algorithm.

To shed light on the ingredients for convergence, we discuss three examples. They show the importance of the special refinement of Theorem 3.1 with an interior node to obtain an error reduction, as well as the necessity of controlling data oscillation.

Example 3.6 (interior node 1). This example shows the necessity of creating an interior node inside each refined triangle. Consider problem (1.2) with $A=I d, f \equiv 1$, and $\Omega=(0,1) \times(0,1)$. Let $\left\{(0,0),(1,0),(1,1),(0,1),\left(\frac{1}{2}, \frac{1}{2}\right)\right\}$ be the set of vertices of $\mathcal{T}_{0}$ (see Figure 3.1, left).

Let $\phi_{1}$ be the nodal basis function of $\mathbb{V}_{0}$ that corresponds to the node $\left(\frac{1}{2}, \frac{1}{2}\right)$. Then it is easily seen that the finite element solution $u_{0}$ is $u_{0}=\frac{1}{12} \phi_{1}$. Let $\mathcal{T}_{1}$ be the grid obtained from $\mathcal{T}_{0}$ by performing two bisections on each triangle of $\mathcal{T}_{0}$ using the newest-vertex bisection and assuming that $\left(\frac{1}{2}, \frac{1}{2}\right)$ is the newest vertex on the initial grid (see Figure 3.1, middle). This is the standard refinement performed in [8] and does not lead to an interior node in the refined elements. Then we get a set of five nodes, and the finite element solution $u_{1}$ on $\mathcal{T}_{1}$ solves a simple $5 \times 5$ system which satisfies $u_{1}=u_{0}$, as can be seen in Figure 3.1. Since $u-u_{1}=u-u_{0}$, we conclude that without one interior node in at least one triangle, no error reduction is obtained even when $\operatorname{osc}\left(f, \mathcal{T}_{0}\right)=0$ and $\left\|u-u_{0}\right\|_{\Omega}>0$. The presence of interior nodes (with respect to $\mathcal{T}_{0}$ ) in the refinement $\mathcal{T}_{2}$ of $\mathcal{T}_{1}$ yields a change of solution values (see Figure 3.1, right).

Example 3.7 (interior node 2). At first sight, it may seem that the situation of Example 3.6 may occur only at the first refinement step. This example shows that such a situation can also happen at any refinement step $n$.

Fix $n \in \mathbb{N}$ and consider (1.2) with $A=I d, \Omega=(0,1)^{2}$, and $f$ given by

$$
f(x)=\left\{\begin{aligned}
1 & \text { if } x \in\left(i 2^{-n},(i+1) 2^{-n}\right) \times\left(j 2^{-n},(j+1) 2^{-n}\right) \text { and } i+j \text { odd } \\
-1 & \text { otherwise }
\end{aligned}\right.
$$



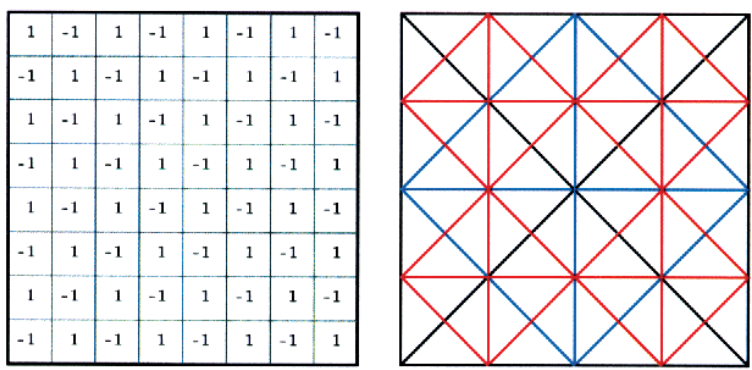

$$
\mathcal{T}_{0}-\mathcal{T}_{1}-\mathcal{T}_{2}-
$$

Fig. 3.2 Example 3.7: Checkerboard function $f$ for $n=3$ (left), and grids $\mathcal{T}_{k}$ for $k=0,1,2$ (right).
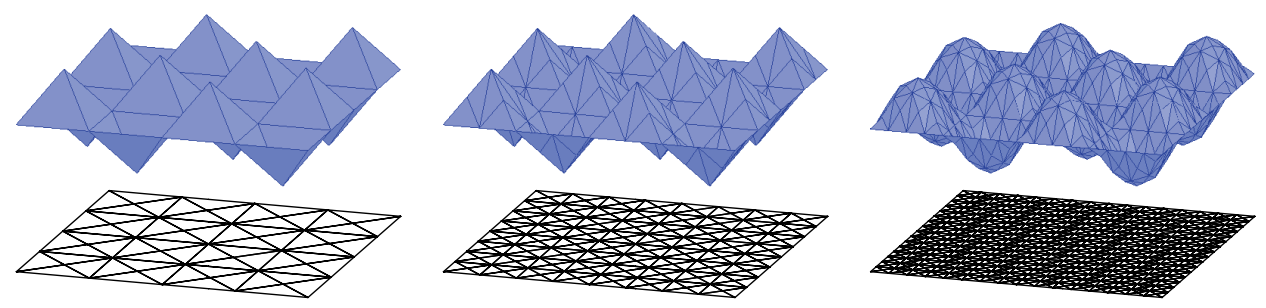

Fig. 3.3 Example 3.7: Finite element solutions $u_{2}, u_{3}, u_{4}$ and meshes $\mathcal{T}_{2}, \mathcal{T}_{3}, \mathcal{T}_{4}$ with $n=2$. Since $u_{2}=u_{3} \neq u_{4}$, error reduction may fail to hold at any adaptive loop, not just at the first one.

see Figure 3.2. Then, if we start with $\mathcal{T}_{0}$ equal to the grid $\mathcal{T}_{0}$ of Example 3.6, and $\phi_{1}$ also as in Example 3.6, we have that $\phi_{1}$ is orthogonal to $f$ and consequently $u_{0} \equiv 0$. If we now define recursively $\mathcal{T}_{k+1}, k=0,1, \ldots$, as the grid that results from $\mathcal{T}_{k}$ by performing two newest-vertex bisections on every triangle (see Figure 3.2), we will have $u_{k} \equiv 0$ for $k=0,1, \ldots, n-1$, due to the fact that $f$ is orthogonal to the basis functions of $\mathcal{T}_{k}$ for $k=0,1, \ldots, n-1$.

For $k=n$ the solution $u_{n}$ will not be zero anymore, but it will be zero along the lines where $f$ changes sign due to the symmetry of the problem, and the same will happen with $u_{n+1}$ (see Figure 3.3). Then, if we observe $u_{n}$ and $u_{n+1}$ in a fixed square where $f$ is constant, they behave exactly as $u_{0}$ and $u_{1}$ do in Example 3.6, and consequently $u_{n}=u_{n+1}$, which means that the error does not decrease, even when the oscillation $\operatorname{osc}\left(f, \mathcal{T}_{n}\right)$ is zero. Figure 3.3 depicts this situation.

Example 3.8 (data oscillation). This example shows that if the data oscillation $\operatorname{osc}\left(f, \mathcal{T}_{H}\right)$ is not small, then, even introducing an interior node on all elements, the error may not decrease. To see this, consider Example 3.7 for some fixed large $n \in \mathbb{N}$. Observe now that if we obtain $\mathcal{T}_{k+1}$ by performing three bisections on all the elements of $\mathcal{T}_{k}$, then three new nodes are created on the edge opposite to the newest vertex in addition to an interior node per element (see Figure 3.4). Even though this refinement is stronger than required by Theorem 3.1 in each step, the solutions $u_{k}$ will all be zero for $k<2 n / 3$.

We conclude from Examples 3.6 and 3.7 that the interior node is necessary to obtain an error decrease, and from Example 3.8 that this may not be sufficient if the mesh does not resolve oscillation of data. Therefore, in order to obtain an asymptoti- 


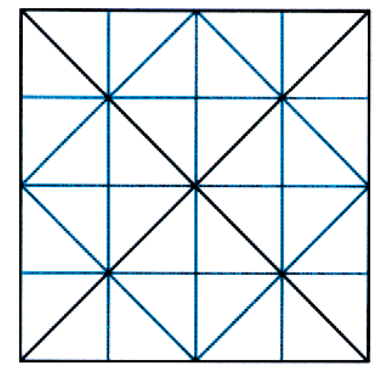

$\mathcal{T}_{0}-\mathcal{T}_{1}-$

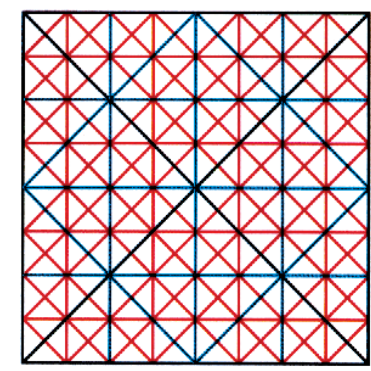

$\mathcal{T}_{1}-\mathcal{T}_{2}-$

Fig. 3.4 Resulting grid $\mathcal{T}_{1}$ (left) and $\mathcal{T}_{2}$ (right) after performing three bisections on each element of $\mathcal{T}_{0}$ and $\mathcal{T}_{1}$, respectively.

cally convergent sequence of discrete solutions, we must readjust the mesh to resolve $\operatorname{osc}\left(f, \mathcal{T}_{H}\right)$ according to a decreasing tolerance. The following simple results guarantee that this is always possible. The first lemma considers the worst scenario situation of $f$ just in $L^{2}(\Omega)$, whereas the second lemma addresses the case of $f$ piecewise smooth.

Lemma 3.9. Let $0<\gamma<1$ be the reduction factor of element size associated with one refinement step. Given $0<\hat{\theta}<1$, let $\hat{\alpha}:=\left(1-\left(1-\gamma^{2}\right) \hat{\theta}^{2}\right)^{1 / 2}$. Let $\hat{\mathcal{T}}_{H}$ be a subset of $\mathcal{T}_{H}$ such that

$$
\operatorname{osc}\left(f, \hat{\mathcal{T}}_{H}\right) \geq \hat{\theta} \operatorname{osc}\left(f, \mathcal{T}_{H}\right) .
$$

If $\mathcal{T}_{h}$ is a triangulation obtained from $\mathcal{T}_{H}$ by refining at least every element in $\hat{\mathcal{T}}_{H}$, then the following data oscillation reduction occurs:

$$
\operatorname{osc}\left(f, \mathcal{T}_{h}\right) \leq \hat{\alpha} \operatorname{osc}\left(f, \mathcal{T}_{H}\right) .
$$

Proof. Let $T \in \mathcal{T}_{h}$ be an element contained in $\hat{T} \in \hat{\mathcal{T}}_{H}$. Since $f_{T}=|T|^{-1} \int_{T} f$ is the $L^{2}$-projection of $f$ onto the space of piecewise constants over $T$, we have

$$
\left\|f-f_{T}\right\|_{T} \leq\left\|f-f_{\hat{T}}\right\|_{T} .
$$

Since $h_{T} \leq \gamma h_{\hat{T}}$, we discover that

$$
\begin{aligned}
\operatorname{osc}\left(f, \mathcal{T}_{h}\right)^{2} & =\sum_{T \in \mathcal{T}_{h}} h_{T}^{2}\left\|f-f_{T}\right\|_{T}^{2} \\
& \leq \gamma^{2} \sum_{\hat{T} \in \hat{\mathcal{T}}_{H}} h_{\hat{T}}^{2}\left\|f-f_{\hat{T}}\right\|_{\hat{T}}^{2}+\sum_{T \in \mathcal{T}_{H} \backslash \hat{\mathcal{T}}_{H}} h_{T}^{2}\left\|f-f_{T}\right\|_{T}^{2} \\
& =\left(\gamma^{2}-1\right) \operatorname{osc}\left(f, \hat{\mathcal{T}}_{H}\right)^{2}+\operatorname{osc}\left(f, \mathcal{T}_{H}\right)^{2} \leq \hat{\alpha}^{2} \operatorname{osc}\left(f, \mathcal{T}_{H}\right)^{2} .
\end{aligned}
$$

Lemma 3.10. Let $f$ be piecewise $H^{s}$ for $0<s \leq 1$ over the initial mesh, where $H^{s}$ stands for the space of functions $f$ with fractional derivative $D^{s} f$ of order $s$ in $L^{2}$. Let data oscillation be redefined by

$$
\operatorname{osc}\left(f, \mathcal{T}_{h}\right):=\left(\sum_{T \in \mathcal{T}_{h}} h_{T}^{2+2 s}\left\|D^{s} f\right\|_{T}^{2}\right)^{1 / 2} .
$$


Let $\hat{\alpha}:=\left(1-\left(1-\gamma^{2+2 s}\right)^{2}\right)^{1 / 2}$, where $\gamma$ and $\hat{\theta}$ are defined in Lemma 3.9. If $\mathcal{T}_{h}$ is as in Lemma 3.9, then

$$
\operatorname{osc}\left(f, \mathcal{T}_{h}\right) \leq \hat{\alpha} \operatorname{osc}\left(f, \mathcal{T}_{H}\right) .
$$

Proof. It suffices to use the fact that $h_{T} \leq \gamma h_{\hat{T}}$ for all $T \in \mathcal{T}_{h}$ contained in $\hat{T} \in \hat{\mathcal{T}}_{H}$ and argue as in Lemma 3.9.

We point out that $\gamma$ depends only on the minimal angle of the initial mesh, and that $\gamma \leq 1 / 2$ in two dimensions provided one interior node is created per marked element; we refer to Figure 5.1 of section 5 . This implies that the reduction rate squared of Lemma 3.10 is $\hat{\alpha}^{2} \leq 1-15 \hat{\theta}^{2} / 16 \approx 1-\hat{\theta}^{2}$ in two dimensions provided $f$ is piecewise $H^{1}$.

Taking into account that a subset $\hat{\mathcal{T}}_{H}$ of $\mathcal{T}_{H}$ is already selected by Marking Strategy E, the following procedure, in conjunction with Lemma 3.9 or 3.10, guarantees a data oscillation decrease by a factor $\hat{\alpha}<1$.

Marking Strategy $\mathbf{D}$
Given a parameter $0<\hat{\theta}<1$ and the subset $\hat{\mathcal{T}}_{H} \subset \mathcal{T}_{H}$ produced by
Marking Strategy E:
1. Enlarge $\hat{\mathcal{T}}_{H}$ such that
\[ \operatorname{osc}\left(f, \hat{\mathcal{T}}_{H}\right) \geq \hat{\theta} \operatorname{osc}\left(f, \mathcal{T}_{H}\right) \]
2. Mark all elements in $\hat{\mathcal{T}}_{H}$ for refinement.

In light of Theorem 3.1, Examples 3.6, 3.7, and 3.8, and Lemmas 3.9 and 3.10, we are now in the position to formulate Algorithm $\mathrm{C}$ and prove its asymptotic convergence.

\section{Convergent Algorithm C}

Choose parameters $0<\theta, \hat{\theta}<1$.

1. Pick up any initial mesh $\mathcal{T}_{0}$ such that $A$ is piecewise constant over $\mathcal{T}_{0}$ and set $k:=0$.

2. Solve the system on $\mathcal{T}_{k}$ for the discrete solution $u_{k}$.

3. Compute the local indicators $\eta_{S}$.

4. Construct $\hat{\mathcal{T}}_{k} \subset \mathcal{T}_{k}$ by Marking Strategy $\mathrm{E}$ and parameter $\theta$.

5. Enlarge $\hat{\mathcal{T}}_{k}$ by Marking Strategy $\mathrm{D}$ and parameter $\hat{\theta}$.

6. Let $\mathcal{T}_{k+1}$ be a refinement of $\mathcal{T}_{k}$ satisfying the interior node property (3.1).

7. Set $k:=k+1$ and go to Step 2 .

Theorem 3.11 (convergence). For $0<\theta, \hat{\theta}<1$, let $0<\alpha<1$ be given by Theorem 3.1 and $0<\hat{\alpha}<1$ by Lemma 3.9 or 3.10 . Let $\alpha_{0}:=\max (\alpha, \hat{\alpha})$. Then for every $\beta$ such that $\alpha_{0}<\beta<1$, the sequence $\left\{u_{k}\right\}_{k \in \mathbb{N}_{0}}$ of finite element solutions produced by Algorithm $C$ satisfies

$$
\left\|u-u_{k}\right\|_{\Omega} \leq C_{0} \beta^{k}
$$

with

$$
C_{0}:=\left\|u-u_{0}\right\|_{\Omega}+\frac{1}{\beta-\alpha_{0}} \operatorname{osc}\left(f, \mathcal{T}_{0}\right) .
$$


Proof. We first apply Lemma 3.9 recursively to get

$$
\operatorname{osc}\left(f, \mathcal{T}_{k}\right) \leq \hat{\alpha} \operatorname{osc}\left(f, \mathcal{T}_{k-1}\right) \leq \cdots \leq \hat{\alpha}^{k} \operatorname{osc}\left(f, \mathcal{T}_{0}\right) .
$$

We then set $e_{k}:=\left\|u-u_{k}\right\|_{\Omega}$ and utilize Theorem 3.1 to deduce

$$
e_{k+1} \leq \alpha e_{k}+\operatorname{osc}\left(f, \mathcal{T}_{k}\right) \leq \alpha e_{k}+\hat{\alpha}^{k} \operatorname{osc}\left(f, \mathcal{T}_{0}\right),
$$

which by recursion implies

$$
e_{k+1} \leq \alpha^{k+1} e_{0}+\operatorname{osc}\left(f, \mathcal{T}_{0}\right) \sum_{j=0}^{k} \alpha^{j} \hat{\alpha}^{k-j} .
$$

Since $\alpha \leq \alpha_{0}$ and $\hat{\alpha}<\beta$, we obtain the estimate

$$
\sum_{j=0}^{k} \alpha^{j} \hat{\alpha}^{k-j} \leq \beta^{k} \sum_{j=0}^{k}\left(\frac{\alpha_{0}}{\beta}\right)^{j} \leq \beta^{k} \frac{1}{1-\frac{\alpha_{0}}{\beta}}=\beta^{k+1} \frac{1}{\beta-\alpha_{0}},
$$

from which the assertion follows immediately.

Remark 3.12. Algorithm C is fully practical in that it needs only parameters $\theta, \hat{\theta}$. The unknown constants $\alpha$ and $\hat{\alpha}$ are not explicitly needed by Algorithm $\mathrm{C}$, but they dictate the convergence rate $\beta$, which improves upon and is more explicit than the original value of $\beta$ in [16]; observe that $\beta$ can be as close to $\alpha_{0}$ as desired! Moreover, if $\alpha \neq \hat{\alpha}$, we have that $\alpha_{0}>\alpha_{1}:=\min (\alpha, \hat{\alpha})$, and in this case the assertion of the theorem holds even with $\beta=\alpha_{0}$, taking $C_{0}=\left\|u-u_{0}\right\|_{\Omega}+\frac{1}{\alpha_{0}-\alpha_{1}} \operatorname{osc}\left(f, \mathcal{T}_{0}\right)$.

Remark 3.13. Stopping the sequence $\left\{u_{k}\right\}_{k \in \mathbb{N}_{0}}$ to achieve an error tolerance is the only instance that requires the constant $C_{1}$ of Lemma 2.1. However, this difficulty is typical of residual-type estimators and is thus customary in the literature.

Remark 3.14. Since all existing a posteriori error estimators for (1.2) are equivalent to the residual estimator $\eta\left(u_{H}, \mathcal{S}_{H}\right)$, perhaps up to data oscillation, Theorem 3.11 extends to them. We refer especially to [17] because it deals with a new star estimator with excellent effectivity indices and shows convergence of adaptive loops without ever resorting to comparisons with $\eta\left(u_{H}, \mathcal{S}_{H}\right)$.

Remark 3.15. In view of Lemma 3.10, and since we only have to expand the set of already marked elements $\hat{\mathcal{T}}_{H}$ by Marking Strategy D, we expect data oscillation to yield rather minor additional refinement to step 4 . This is confirmed by the numerical experiments of section 5.4 in both two and three dimensions, and provides some solid theoretical grounds for the convergence of adaptive strategies which disregard data oscillation altogether.

4. Error Reduction. In this section we prove Theorem 3.1. The following orthogonality result is essential and gives us an idea of how to proceed.

LEMma 4.1. If $\mathcal{T}_{h}$ is a local refinement of $\mathcal{T}_{H}$, such that $\mathbb{V}^{H} \subset \mathbb{V}^{h}$, the following relation holds:

$$
\left\|u-u_{h}\right\|_{\Omega}^{2}=\left\|u-u_{H}\right\|_{\Omega}^{2}-\left\|u_{H}-u_{h}\right\|_{\Omega}^{2} .
$$

Proof. By Galerkin orthogonality, $a\left(u-u_{h}, v\right)=0$ for all $v \in \mathbb{V}_{0}^{h}$, whence $u_{h}-$ $u_{H} \in \mathbb{V}_{0}^{h}$ is perpendicular to $u-u_{h}$. Therefore, since $u-u_{H}=\left(u-u_{h}\right)+\left(u_{h}-u_{H}\right)$, the assertion (4.1) follows from the Pythagoras theorem.

We can see from this lemma that the error reduction is exactly $\left\|u_{H}-u_{h}\right\|_{\Omega}^{2}$. In order to guarantee that the error decreases a fixed proportion of the current error, we 


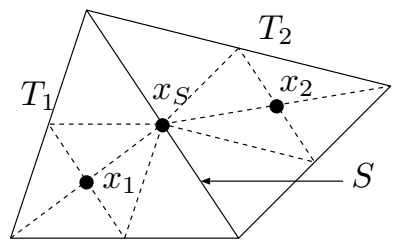

Fig. 4.I Example of a refined two-element patch $\omega_{S}=T_{1} \cup T_{2}$ in two dimensions.
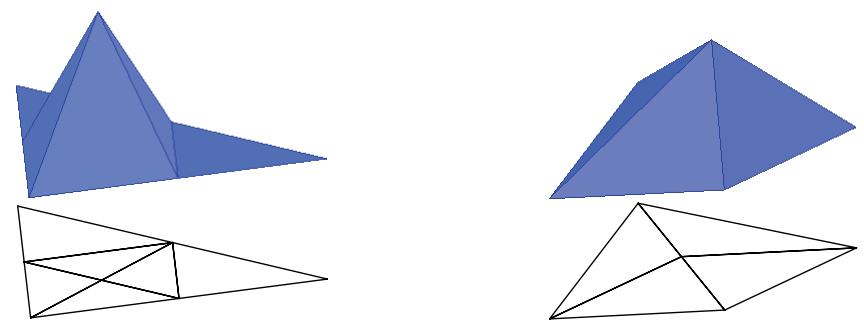

Fig. 4.2 Discrete bubble functions $\varphi_{1}, \varphi_{S}$ associated with the interior nodes $x_{1}, x_{S}$ of Figure 4.1.

have to bound $\left\|u_{H}-u_{h}\right\|_{\Omega}^{2}$ from below by $\left\|u-u_{H}\right\|_{\Omega}^{2}$. In view of Lemma 2.2 , this reduces to showing a lower bound in terms of $\eta_{H}^{2}$. The following lemma establishes a local lower bound for the error decrease in terms of the local error indicators. This result will be needed, in conjunction with Lemma 2.1, to prove a global lower bound of the error decrease in terms of the current error.

LEMmA 4.2. Let $\mathcal{T}_{H}$ be a triangulation of $\Omega$, and let $\hat{\mathcal{T}}_{H}$ and $\hat{\mathcal{S}}_{H}$ be as defined in Marketing Strategy E. Let $\mathcal{T}_{h}$ be a refinement of $\mathcal{T}_{H}$ satisfying (3.1). Then there exists a constant $C_{4}$, depending only on the minimum angle and $c_{a}, C_{a}$, such that

$$
\eta_{S}^{2} \leq C_{4}\left(\left\|u_{h}-u_{H}\right\|_{\omega_{S}}^{2}+\left\|H\left(f-f_{H}\right)\right\|_{\omega_{S}}^{2}\right) \quad \forall S \in \hat{\mathcal{S}}_{H}
$$

Before getting into the proof of this lemma, observe that this result is similar to the result of Lemma 2.2. Their main differences are that here we get a lower bound for $\left\|u_{h}-u_{H}\right\|_{\omega_{S}}$ instead of $\left\|u-u_{H}\right\|_{\omega_{S}}$, and that $f_{H}$ stands for a piecewise constant approximation of $f$; i.e., $f_{H}$ has only one degree of freedom in each triangle $T \in \mathcal{T}_{H}$.

Proof of Lemma 4.2. The proof consists of several steps. We first introduce the nodal basis functions $\varphi_{S}, \varphi_{1}$, and $\varphi_{2}$ of $\mathbb{V}_{0}^{h}$ associated with the interior nodes $x_{S}$ of $S$ and $x_{1}, x_{2}$ of $T_{1}$ and $T_{2}$, respectively; here $T_{1}$ and $T_{2}$ denote the two elements of $\mathcal{T}_{H}$ sharing $S$ (see Figure 4.1). Note that the existence of $x_{1}, x_{2}$, and $x_{S}$ is guaranteed by step 6 of Algorithm C.

Integrating by parts in $\omega_{S}$, we deduce the following equation for $u_{h}-u_{H}$ :

$$
a\left(u_{h}-u_{H}, \varphi\right)=\langle f, \varphi\rangle_{\omega_{S}}+\left\langle J_{S}, \varphi\right\rangle_{S}=\left\langle f_{H}, \varphi\right\rangle_{\omega_{S}}+\left\langle J_{S}, \varphi\right\rangle_{S}+\left\langle f-f_{H}, \varphi\right\rangle_{\omega_{S}}
$$

for all $\varphi \in \mathbb{V}_{0}^{h} \cap H_{0}^{1}\left(\omega_{S}\right)$. We choose the bubble functions $\varphi_{S}, \varphi_{1}, \varphi_{2} \in \mathbb{V}_{0}^{h} \cap H_{0}^{1}\left(\omega_{S}\right)$, properly scaled, as test functions in this error representation formula (see Figure 4.2).

Since $f_{H}$ is piecewise constant, we have $\varphi=f_{H} \varphi_{i} \in \mathbb{V}_{0}^{h} \cap H_{0}^{1}\left(T_{i}\right)(i=1,2)$ and thus obtain by the localization due to $\varphi_{i}$ and scaling arguments 


$$
\begin{aligned}
c\left\|f_{H}\right\|_{T_{i}}^{2} & =\left\langle f_{H}, f_{H} \varphi_{i}\right\rangle_{T_{i}}=a\left(u_{h}-u_{H}, f_{H} \varphi_{i}\right)-\left\langle f-f_{H}, f_{H} \varphi_{i}\right\rangle_{T_{i}} \\
& \leq\left\|u_{h}-u_{H}\right\|_{T_{i}}\left\|f_{H} \varphi_{i}\right\|_{T_{i}}+\left\|f-f_{H}\right\|_{T_{i}}\left\|f_{H} \varphi_{i}\right\|_{T_{i}} .
\end{aligned}
$$

Using the continuity of the bilinear form, namely, $\left\|f_{H} \varphi_{i}\right\|_{T_{i}} \leq C_{a}\left\|f_{H} \nabla \varphi_{i}\right\|_{T_{i}}$, together with the inverse estimate $\left\|f_{H} \nabla \varphi_{i}\right\|_{T_{i}} \leq C h_{S}^{-1}\left\|f_{H}\right\|_{T_{i}}$, we conclude for $i=1,2$

$$
h_{S}\|f\|_{T_{i}} \leq h_{S}\left\|f_{H}\right\|_{T_{i}}+h_{S}\left\|f-f_{H}\right\|_{T_{i}} \leq C\left(\left\|u_{h}-u_{H}\right\|_{T_{i}}+h_{S}\left\|f-f_{H}\right\|_{T_{i}}\right) .
$$

We proceed analogously for the jump $J_{S}$. Since $J_{S}$ is also constant we have $\varphi=J_{S} \varphi_{S} \in \mathbb{V}_{0}^{h} \cap H_{0}^{1}\left(\omega_{S}\right)$ and similar scaling arguments imply

$$
\begin{aligned}
c\left\|J_{S}\right\|_{S}^{2} & =\left\langle J_{S}, J_{S} \varphi_{S}\right\rangle_{S}=a\left(u_{h}-u_{H}, J_{S} \varphi\right)-\left\langle f, J_{S} \varphi\right\rangle_{\omega_{S}} \\
& \leq C\left(\left\|u_{h}-u_{H}\right\|_{\omega_{S}}+h_{S}\|f\|_{\omega_{S}}\right) h_{S}^{-1}\left\|J_{S}\right\|_{\omega_{S}} .
\end{aligned}
$$

Applying the scaling $\left\|J_{S}\right\|_{\omega_{S}} \leq h_{S}^{\frac{1}{2}}\left\|J_{S}\right\|_{S}$ in combination with (4.3), we conclude

$$
h_{S}^{\frac{1}{2}}\left\|J_{S}\right\|_{S} \leq C\left(\left\|u_{h}-u_{H}\right\|_{\omega_{S}}+h_{S}\left\|f-f_{H}\right\|_{\omega_{S}}\right),
$$

which finishes the proof.

Now we can state and prove a global lower bound for the error reduction.

Corollary 4.3. Let $\mathcal{T}_{H}$ and $\mathcal{T}_{h}$ be triangulations of $\Omega$ satisfying (3.1). Then we have the following global lower bound for the error reduction:

$$
\left\|u_{h}-u_{H}\right\|_{\Omega}^{2} \geq \frac{\theta^{2}}{(d+1) C_{4} C_{1}}\left\|u-u_{H}\right\|_{\Omega}^{2}-\operatorname{osc}\left(f, \mathcal{T}_{H}\right)^{2} .
$$

Proof. By Lemma 4.2 and step 1 of Marking Strategy E we have

$$
\begin{aligned}
\theta^{2} \eta\left(u_{H}, \mathcal{S}_{H}\right)^{2} & \leq \eta\left(u_{H}, \hat{\mathcal{S}}_{H}\right)^{2} \leq C_{4} \sum_{S \in \hat{\mathcal{S}}_{H}}\left(\left\|u_{h}-u_{H}\right\|_{\omega_{S}}^{2}+\left\|H\left(f-f_{H}\right)\right\|_{\omega_{S}}^{2}\right) \\
& \leq(d+1) C_{4}\left(\left\|u_{h}-u_{H}\right\|_{\Omega}^{2}+\left\|H\left(f-f_{H}\right)\right\|_{\Omega}^{2}\right) .
\end{aligned}
$$

Hence,

$$
\left\|u_{h}-u_{H}\right\|_{\Omega}^{2} \geq \frac{\theta^{2}}{(d+1) C_{4}} \eta\left(u_{H}, \mathcal{S}_{H}\right)^{2}-\left\|H\left(f-f_{H}\right)\right\|_{\Omega}^{2},
$$

and the assertion follows from (1.3) and Lemma 2.1.

Lemmas 4.1, 4.2, and Corollary 4.3 have provided us with the necessary tools to prove Theorem 3.1 .

Proof of Theorem 3.1. In view of Lemma 4.1 and Corollary 4.3, we have

$$
\begin{aligned}
\left\|u-u_{h}\right\|_{\Omega}^{2} & =\left\|u-u_{H}\right\|_{\Omega}^{2}-\left\|u_{H}-u_{h}\right\|_{\Omega}^{2} \\
& \leq\left\|u-u_{H}\right\|_{\Omega}^{2}\left(1-\frac{\theta^{2}}{(d+1) C_{4} C_{1}}\right)+\operatorname{osc}\left(f, \mathcal{T}_{H}\right)^{2},
\end{aligned}
$$

which proves Theorem 3.1 with $\alpha=\sqrt{1-\frac{\theta^{2}}{(d+1) C_{4} C_{1}}}$.

5. Numerical Experiments. We start with a brief discussion of crucial implementation issues, and conclude with three relevant experiments which corroborate convergence of Algorithm $\mathrm{C}$ without mesh preadaptation, produce quasi-optimal meshes, and show a very competitive performance. 


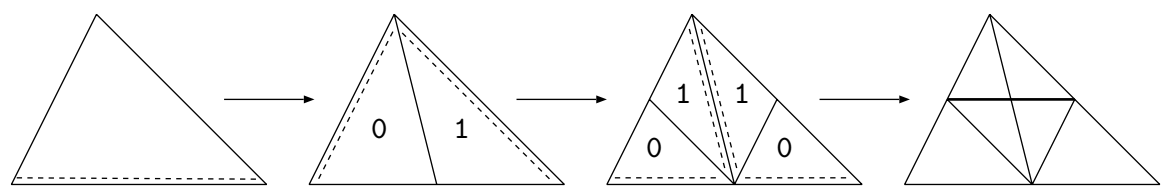

Fig. 5.I Refinement of triangles in two dimensions by newest-vertex bisection. Dashed lines indicate the refinement edges, which are sides opposite to the most recently created nodes.

5.I. Implementation of Algorithm C. Convergent Algorithm C is implemented within the finite element toolbox ALBERT [19, 20]. For the implementation of this algorithm, we have to change the standard adaptive solver for elliptic problems of ALBERT slightly. The following modules are added to, or replace modules of, the standard solver. They are all minor modifications.

Interior Nodes. During the refinement of a marked element we have to ensure that one node in the interior and nodes in all sides of the element are created. ALBERT uses recursive bisectioning refinement: the newest-vertex refinement in two dimensions [15] and the algorithm of Kossaczký [14] in three dimensions. This leads to a sequence of nested grids, which is crucial in obtaining the error orthogonality of Lemma 4.1.

Creating interior nodes is rather easy in two dimensions. First, elements are marked for two bisections and then refined. This already produces a node at the midpoint of each edge. Second, the two grandchildren with index 1 are bisected once more. The whole refinement process is shown in Figure 5.1. The first refinement step may, as usual, involve surrounding elements which are not marked. This is an inevitable effect in order to preserve mesh conformity. The second refinement step is local in that it involves only the two grandchildren with index 1 and does not spread outside them.

In three dimensions it is impossible to perform the second step by dealing only with children of the original tetrahedron. The first step consists of three bisections. In order to obtain the interior nodes, the second step consists of marking some subtetrahedra for two or three additional bisections. This has the spreading effect of creating additional nodes in the edges of the original tetrahedron. For the implementation, we do not split the refinement into two steps, but rather mark a tetrahedron for six bisections which are performed in one step. This creates an interior node in the tetrahedron and interior nodes in all the element faces. Example 5.4 demonstrates experimentally that the additional nodes are not too many and the resulting meshes are still quasi-optimal.

Side-Based Error Estimator. In view of (2.6), we mark sides for refinement. Usually, error indicators are stored element-wise and marking is also done elementwise. These modules are now changed in such a way that the jump residual $\left\|H_{S}^{1 / 2} J_{S}\right\|_{S}$ across a side $S$ and the element residual $\|H f\|_{\omega_{S}}$ of the two adjacent simplices are stored at the side $S$ itself. The marking function thus uses side error indicators $\eta_{S}$ and marks both adjacent elements for refinement.

According to Marking Strategy E, we collect sides in $\hat{\mathcal{S}}_{H}$ with biggest side error indicators $\eta_{S}$ until

$$
\eta\left(u_{H}, \hat{\mathcal{S}}_{H}\right) \geq \theta \eta\left(u_{H}, \mathcal{S}_{H}\right) .
$$

In order to avoid a complicated sorting algorithm for the ordering of the error indicators, we adapt the algorithm proposed in [8]. 
Algorithm 5.1 (marking algorithm). Let $0<\theta, \nu<1$ be given parameters, where $\theta \approx 0.5$ and $\nu \approx 0.1$ :

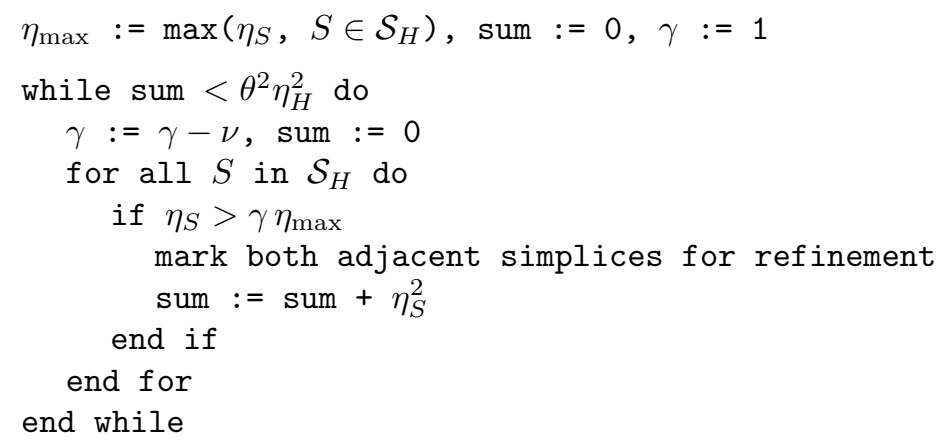

An alternative to side-based marking uses element-stored indicators as follows: an element is marked together with all its neighbors. This leads to a slightly larger number of marked elements but may again be viewed as a looking-ahead strategy. An advantage is that only the marking strategy has to be modified.

Data Oscillation. The last module we supply is a function which marks elements due to data oscillation, i.e., an implementation of Marking Strategy D. For each simplex $T$ we compute the mean value $f_{T}$ of $f$ on $T$ and store the value $\left\|H\left(f-f_{T}\right)\right\|_{T}$. Given $\hat{\theta}$ and $\hat{\mathcal{T}}_{H}$, the set of marked elements by Algorithm 5.1, we enlarge $\hat{\mathcal{T}}_{H}$ in such a way that

$$
\operatorname{osc}\left(f, \hat{\mathcal{T}}_{H}\right) \geq \hat{\theta} \operatorname{osc}\left(f, \mathcal{T}_{H}\right)
$$

holds. If this inequality is satisfied by $\hat{\mathcal{T}}_{H}$, we are done. Otherwise, we use a modification of Algorithm 5.1 to mark additional elements. This is now done element-wise using the oscillation $\left\|H\left(f-f_{T}\right)\right\|_{T}$ of $f$ as an indicator.

Quadrature. Computations involving integrals of (nonconstant) $f$ and the exact solution $u$, such as the right-hand side of the linear system, data oscillation terms, and errors, use a quadrature rule of order 7. Exact integration was used for the construction of stiffness matrices and right-hand sides for constant $f$.

5.2. Example: Crack Problem. We consider the domain $\Omega=\{|x|+|y|<$ $1\} \backslash\{0 \leq x \leq 1, y=0\}$ with a crack and the exact solution $u$ in polar coordinates

$$
u(r, \theta)=r^{\frac{1}{2}} \sin \frac{\theta}{2}-\frac{1}{4} r^{2} .
$$

We solve (1.2) with $A=I$ and $f=1$ and a nonvanishing Dirichlet boundary condition on $\partial \Omega$. We scale the estimator $\eta_{H}$ by the factor 0.25 .

We compare the guaranteed error reduction strategy (GERS) of Dörfler [8] with our convergent strategy (CONV). Using GERS, all marked triangles are only bisected twice; the interior node is not created. Since $f \neq 0$, GERS requires an initial mesh refinement to satisfy the mesh fineness condition; since $f \equiv 1$ this initial mesh refinement is a global refinement. Strategy CONV does not need these preadaptation steps; CONV thus starts from a very coarse mesh.

Figure 5.2 displays a superior performance of CONV in terms of (scaled) computing time (CPU) due to the coarser initial grid for CONV. From Figure 5.3 we realize that the reduction rate $\beta^{k}$ as a function of the iteration number $k$ is comparable for GERS and CONV, the latter being higher because CONV starts from a coarser mesh and thus requires more iterations for the same accuracy. Combining both figures 


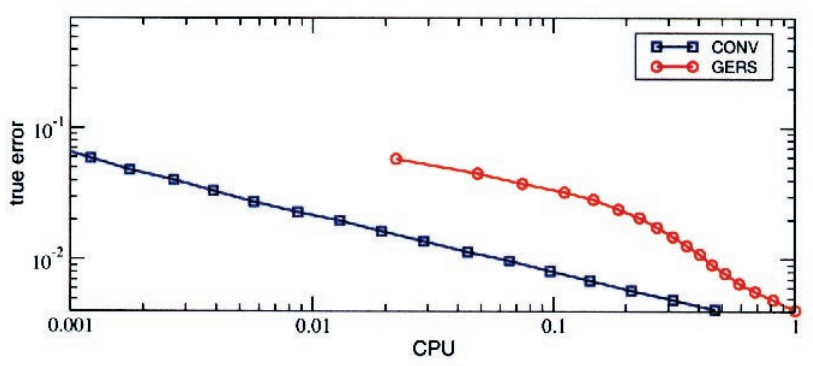

Fig. 5.2 Example 5.2: Comparison of scaled CPU time for GERS and CONV.

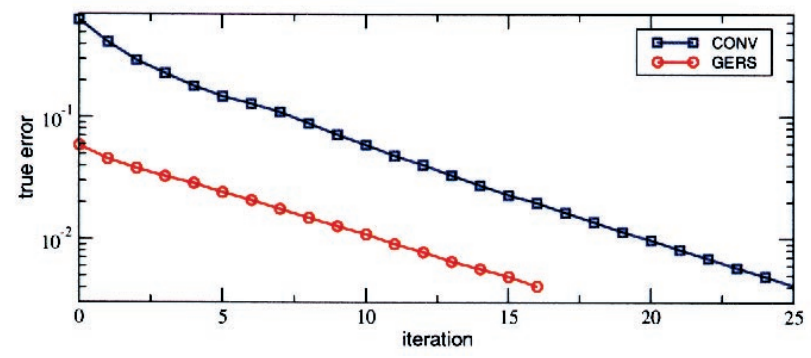

Fig. 5.3 Example 5.2: Comparison of reduction rate $\alpha^{k}$ for GERS and CONV.

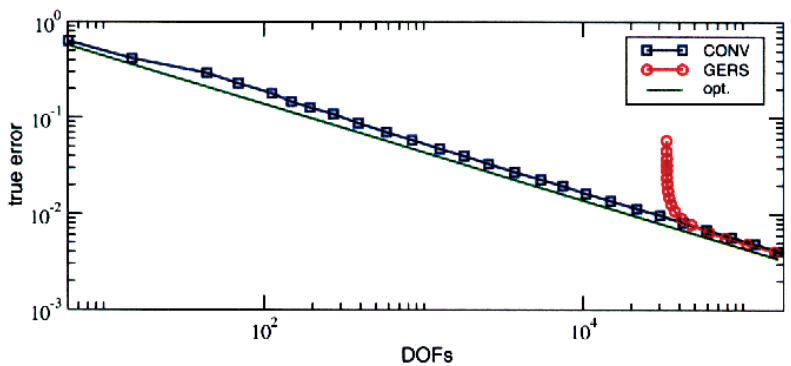

Fig. 5.4 Example 5.2: Quasi-optimality of GERS and CONV. The optimal decay is indicated by the green line with slope $-1 / 2$.

we learn that CONV needs more adaptive iterations to reduce the error below the tolerance 0.05 than GERS but only half the CPU time.

Figure 5.4 is quite revealing. It shows the asymptotic relation $\left\|u-u_{k}\right\|_{\Omega}=C$ $\operatorname{DOFs}(k)^{-1 / 2}$ typical of quasi-optimal meshes in two dimensions and thus of quasioptimal numerical complexity. In the log-log plot the optimal decay of $\left\|u-u_{k}\right\|_{\Omega}$ is a straight line with slope $-1 / 2$. This (green) line is also plotted in Figure 5.4.

Figure 5.5 displays the grid of CONV for iteration 8 , and three zooms at the origin. The rather strong grading of the quasi-optimal partition is quite apparent.

In order to compare the different local mesh-sizes for GERS and CONV due to the preadaptation of GERS, we plot the mesh-sizes along the segment $\{-1<x<0, y=0\}$ 

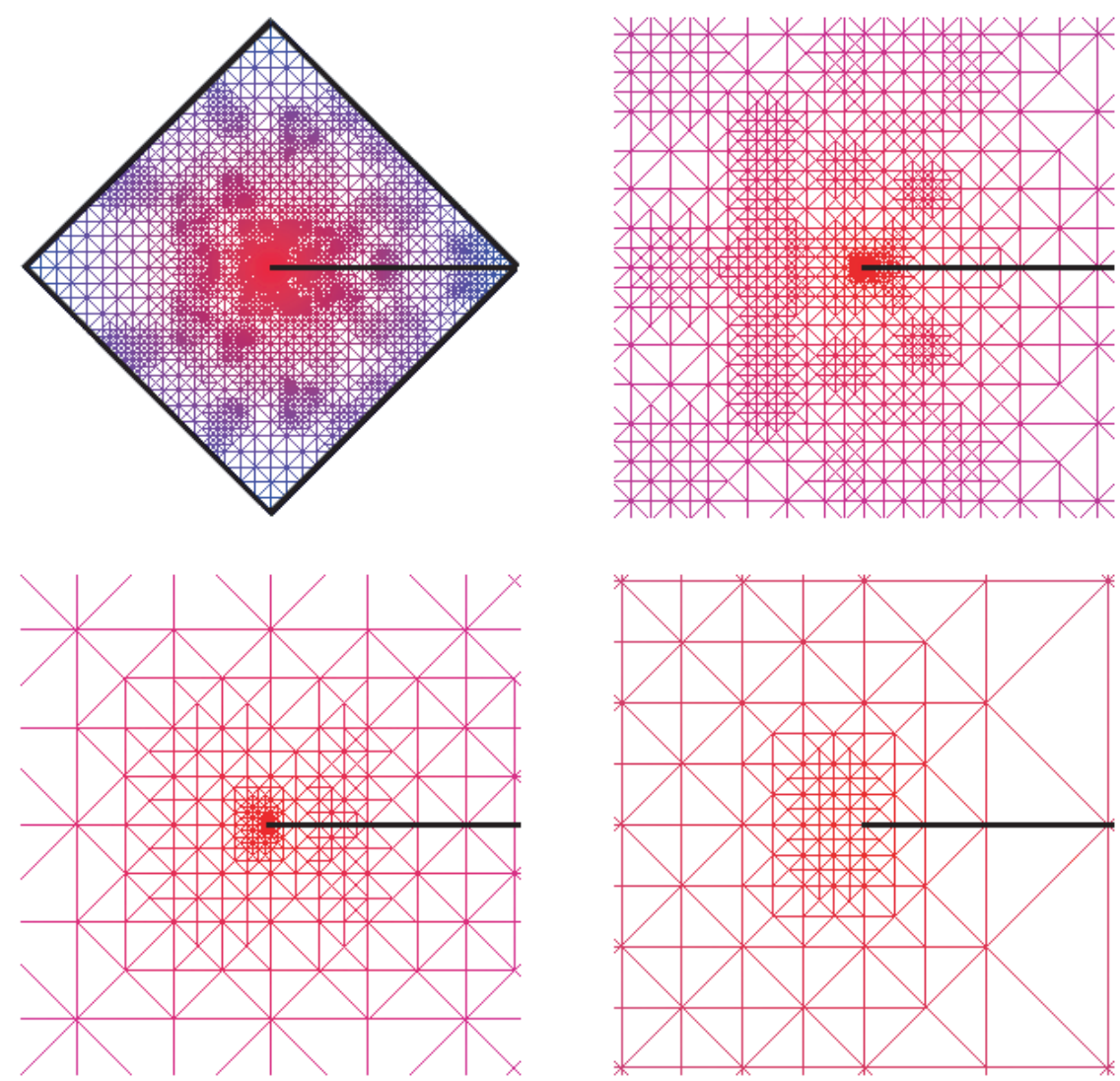

Fig. 5.5 Example 5.2: Adaptive grids for iteration 8 of the CONV strategy: full grid (top left), zooms to $(-0.1,0.1)^{2}$ (top right), $(-0.01,0.01)^{2}$ (bottom left), and $(-0.001,0.001)^{2}$ (bottom right).

in Figure 5.6. We choose the meshes in such a way that the error on the respective meshes is approximately the same for GERS and CONV. The pictures correspond to the meshes in iteration 0 (top left), 4 (top right), 8 (bottom left), and 12 (bottom right) of GERS with errors $\left\|u-u_{k}\right\|_{\Omega} \approx 5.8 \mathrm{e}-02,2.8 \mathrm{e}-02,1.4 \mathrm{e}-02$, and $7.7 \mathrm{e}-03$ and iterations $10,14,18$, and 22 of CONV with errors $5.8 \mathrm{e}-02,2.7 \mathrm{e}-02,1.3 \mathrm{e}-02$, and $6.8 \mathrm{e}-03$. We realize the strong effect of the mesh preadaptation needed by GERS: in contrast to CONV, GERS has to reduce the mesh-size solely near the origin. This corroborates our claim that mesh preadaptation is unnecessary for constant right-hand sides $f$.

Finally, we compare CONV with the maximum strategy (MS), which is not known to converge but is the strategy of choice among practitioners. Figure 5.7 depicts a similar performance of both CONV and MS in terms of degrees of freedom (DOFs).

5.3. Example: Discontinuous Coefficients. We invoke the formulas derived by Kellogg [13] to construct an exact solution of an elliptic problem with piecewise constant coefficients and vanishing right-hand side $f$; data oscillation is thus immaterial. We now write these formulas in the particular case $\Omega=(-1,1)^{2}, A=a_{1} I$ in the first and third quadrants, and $A=a_{2} I$ in the second and fourth quadrants. An exact weak solution $u$ of (1.2) for $f \equiv 0$ is given in polar coordinates by $u(r, \theta)=r^{\gamma} \mu(\theta)$, where 

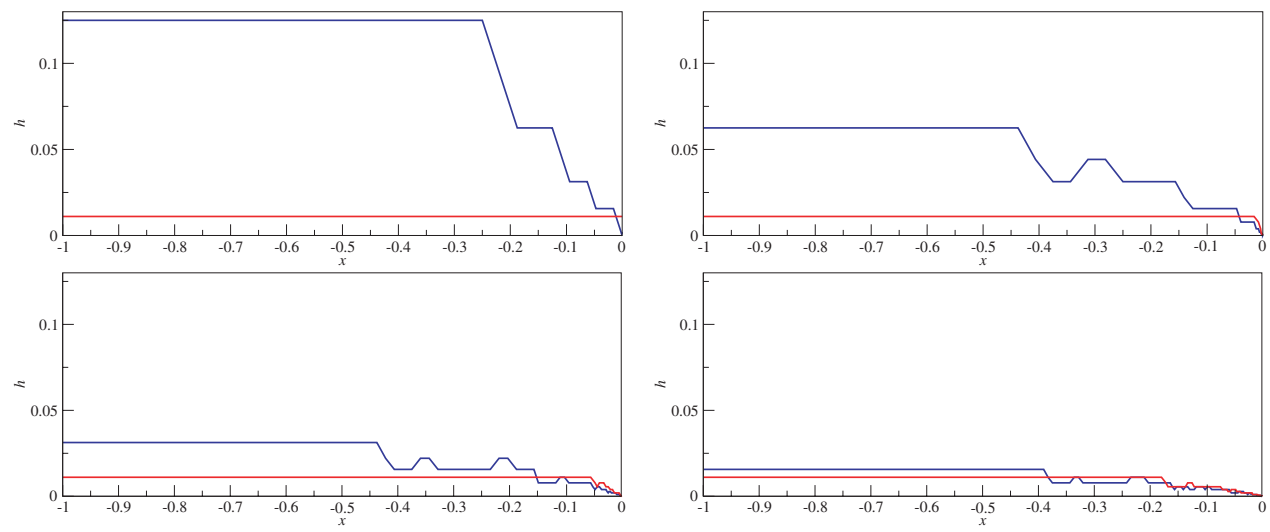

Fig. 5.6 Example 5.2: Comparison of local mesh-sizes $h$ on the line $y=0$ for GERS (red line) and CONV (blue line) on meshes with approximately the same errors $\left\|u-u_{k}\right\|_{\Omega}$.

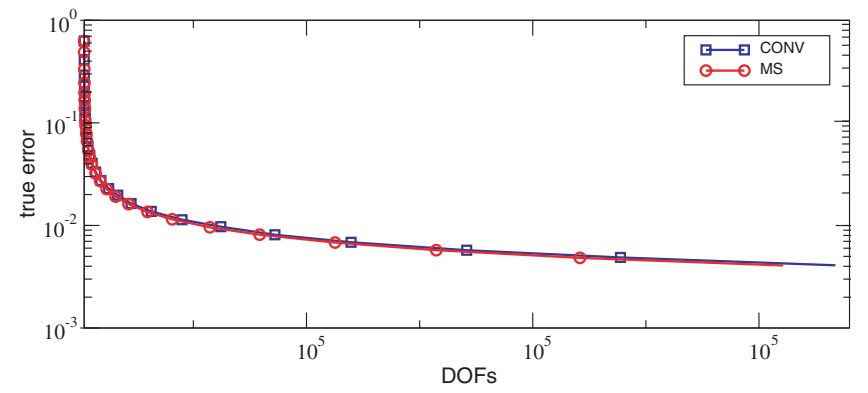

Fig. 5.7 Example 5.2: Comparison of CONV and MS.

$$
\mu(\theta)= \begin{cases}\cos ((\pi / 2-\sigma) \gamma) \cdot \cos ((\theta-\pi / 2+\rho) \gamma) & \text { if } 0 \leq \theta \leq \pi / 2, \\ \cos (\rho \gamma) \cdot \cos ((\theta-\pi+\sigma) \gamma) & \text { if } \pi / 2 \leq \theta \leq \pi, \\ \cos (\sigma \gamma) \cdot \cos ((\theta-\pi-\rho) \gamma) & \text { if } \pi \leq \theta<3 \pi / 2, \\ \cos ((\pi / 2-\rho) \gamma) \cdot \cos ((\theta-3 \pi / 2-\sigma) \gamma) & \text { if } 3 \pi / 2 \leq \theta \leq 2 \pi\end{cases}
$$

and the numbers $\gamma, \rho, \sigma$ satisfy the nonlinear relations

$$
\left\{\begin{array}{l}
R:=a_{1} / a_{2}=-\tan ((\pi / 2-\sigma) \gamma) \cdot \cot (\rho \gamma), \\
1 / R=-\tan (\rho \gamma) \cdot \cot (\sigma \gamma), \\
R=-\tan (\sigma \gamma) \cdot \cot ((\pi / 2-\rho) \gamma), \\
0<\gamma<2, \\
\max \{0, \pi \gamma-\pi\}<2 \gamma \rho<\min \{\pi \gamma, \pi\}, \\
\max \{0, \pi-\pi \gamma\}<-2 \gamma \sigma<\min \{\pi, 2 \pi-\pi \gamma\} .
\end{array}\right.
$$

Since we want to test the algorithm CONV in some worst-case scenario, we choose $\gamma=0.1$, which produces a very singular solution $u$ that is barely in $H^{1}$. We then solve (5.1) for $R, \rho$, and $\sigma$ using Newton's method to obtain

$$
R=a_{1} / a_{2} \cong 161.4476387975881, \quad \rho=\pi / 4, \quad \sigma \cong-14.92256510455152,
$$




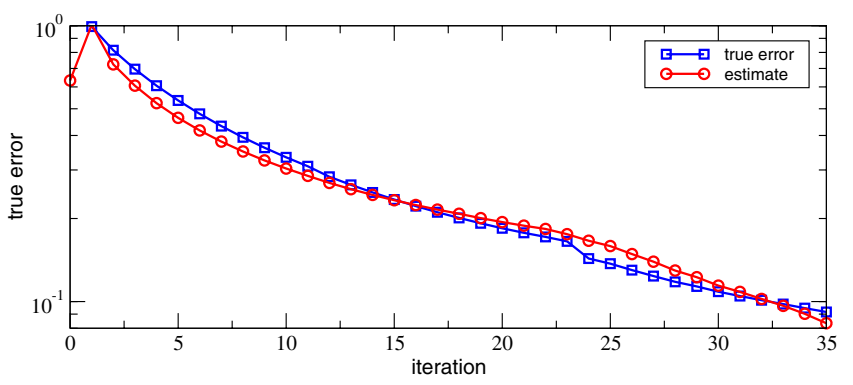

Fig. $\mathbf{5 . 8}$ Example 5.3: Error reduction: estimate and true error.

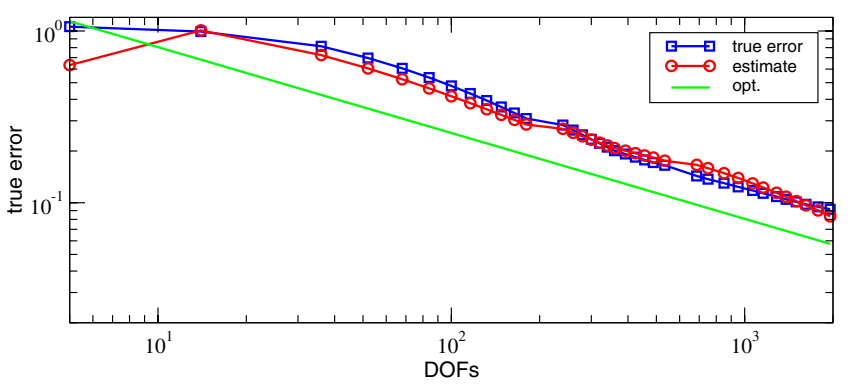

Fig. 5.9 Example 5.3: Quasi-optimality of CONV: estimate and true error. The optimal decay is indicated by the green line with slope $-1 / 2$.

and finally choose $a_{1}=R$ and $a_{2}=1$. A smaller $\gamma$ would lead to a larger ratio $R$, but in principle $\gamma$ may be as close to 0 as desired.

In Figures 5.8-5.9 we see the same behavior of the true error $\left\|u-u_{k}\right\|_{\Omega}$ and the estimator $\eta_{k}$ scaled by the factor 0.05 . Figure 5.9 demonstrates that the grids and associated numerical complexity are quasi-optimal: $\left\|u-u_{k}\right\|_{\Omega}=C \operatorname{DOFs}(k)^{-1 / 2}$ is valid asymptotically (the performance of an optimal method is again indicated by the additional green straight line).

For this problem the grid is highly graded at the origin. It is worth realizing the strength of the singularity at hand in Figure 5.10. We see a mesh with less than 2000 nodes and three zooms at the origin, each obtained with a magnifying factor $10^{3}$, and yet exhibiting a rather strong grading. This is also reflected in Figure 5.11, which depicts the graph of the discrete solution over the underlying mesh: the solution is flatter in the quadrants with $a \approx 161$ although the grid is finer, which accounts for the presence of $a$ in the energy norm. This picture was created using the graphics package GRAPE [12]. This example is much more singular than Example 5.2.

5.4. Example: Variable Source. In Examples 5.2 and 5.3 the source term is constant. It is our purpose now to examine the effect of data oscillation (1.3). To this end, we consider the domain $\Omega=(-1,1)^{d}$ with $d=2,3$ and the exact solution

$$
u(x)=\mathrm{e}^{-10|x|^{2}}
$$

of (1.2) with $A=I$ and nonconstant $f=-\Delta u$. Such an $f$ exhibits a relatively large variation in $\Omega$, and within elements, which forces Algorithm $\mathrm{C}$ to refine additional elements due to data oscillation (step 5 of Algorithm C), not yet marked for refinement 

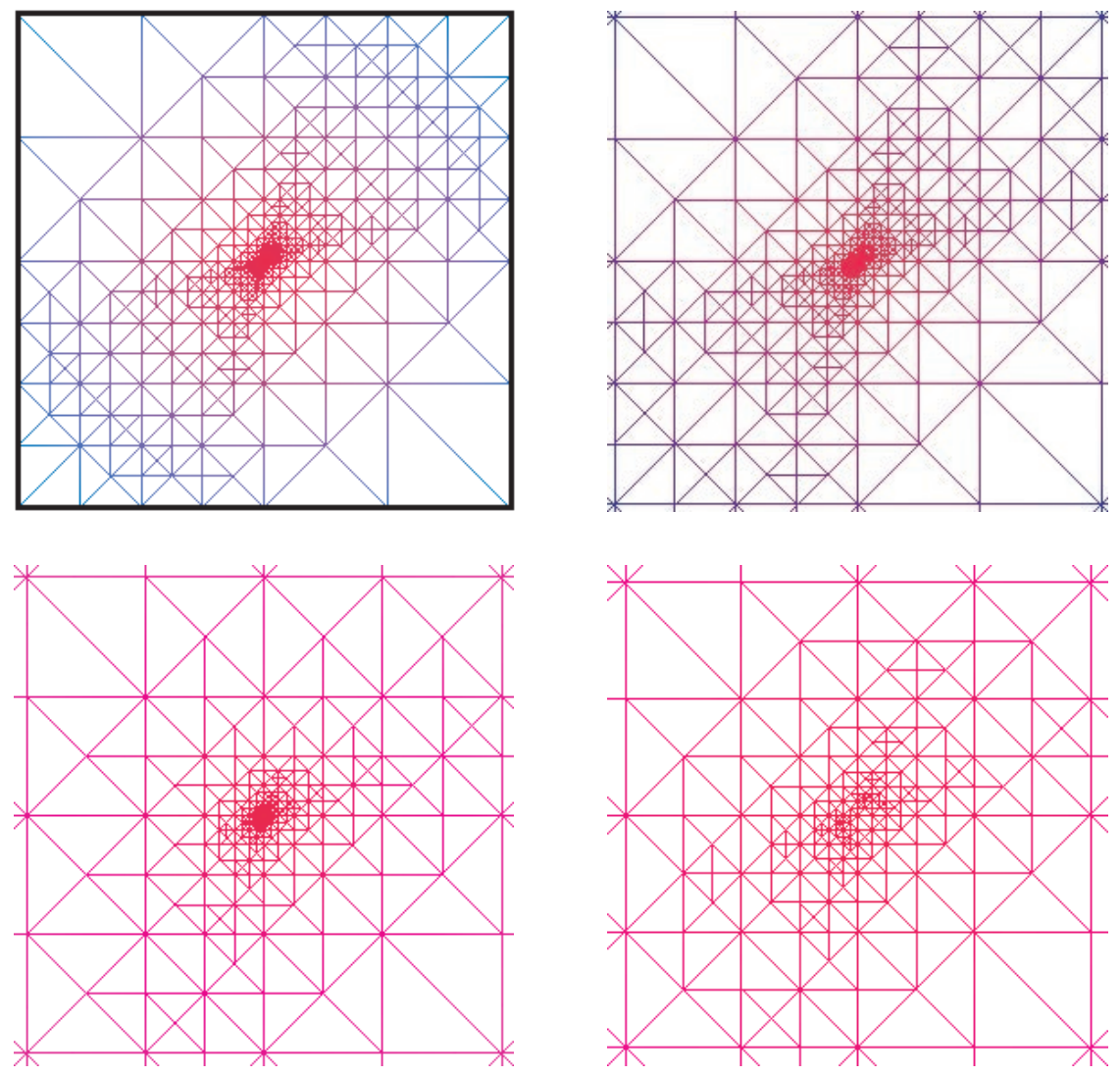

Fig. 5.10 Example 5.3: Final grid (full grid with $<2000$ nodes) $\left(\right.$ top left), zooms to $\left(-10^{-3}, 10^{-3}\right)^{2}$ (top right), $\left(-10^{-6}, 10^{-6}\right)^{2}$ (bottom left), and $\left(-10^{-9}, 10^{-9}\right)^{2}$ (bottom right).

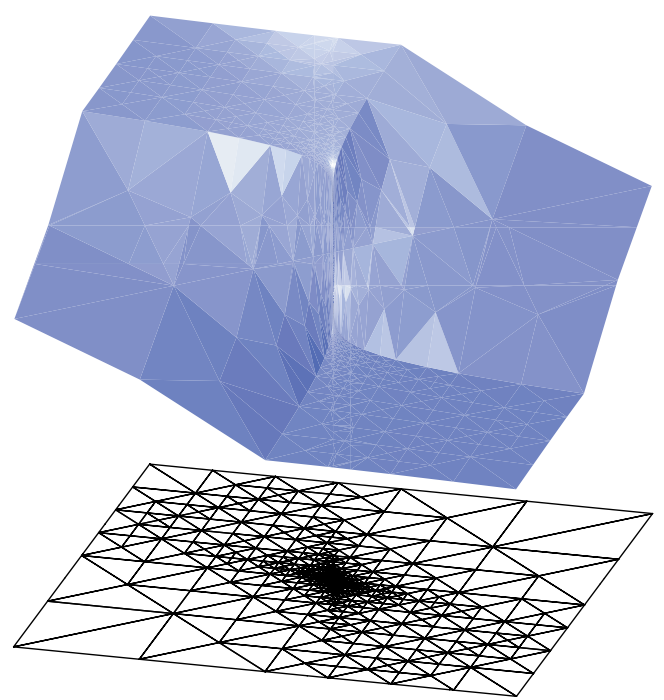

Fig. 5.II Example 5.3: Graph of the discrete solution, which is $\approx r^{0.1}$, and underlying grid. 
Table 5.I Example 5.4: Total number and number of marked elements per iteration in two dimensions (left) and three dimensions (right). Est.: marked elements due to error estimator; Osc.: additionally marked elements to data oscillation.

\begin{tabular}{|r||r|r|r|}
\hline Iter. & Elements & Est. & Osc. \\
\hline \hline 0 & 4 & 8 & 0 \\
1 & 64 & 16 & 16 \\
2 & 704 & 56 & 8 \\
3 & 2256 & 80 & 0 \\
4 & 4208 & 96 & 8 \\
5 & 6624 & 112 & 24 \\
6 & 8752 & 344 & 0 \\
7 & 17512 & 432 & 0 \\
8 & 28368 & 608 & 0 \\
9 & 42896 & 768 & 16 \\
10 & 60216 & 2192 & 0 \\
11 & 113040 & 2296 & 24 \\
12 & 160592 & 3816 & 24 \\
\hline
\end{tabular}

\begin{tabular}{|r||r|r|r|}
\hline Iter. & Elements & Est. & Osc. \\
\hline \hline 0 & 6 & 6 & 0 \\
1 & 384 & 48 & 0 \\
2 & 7776 & 48 & 48 \\
3 & 15936 & 576 & 0 \\
4 & 112320 & 5040 & 0 \\
5 & 860592 & 5136 & 720 \\
6 & 1693536 & 30144 & 0 \\
\hline
\end{tabular}

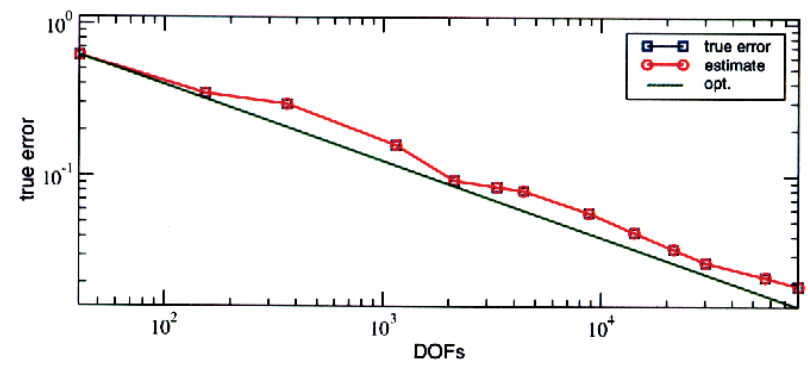

Fig. 5.I2 Example 5.4: Quasi-optimality of CONV: estimate and true error in two dimensions. The optimal decay is indicated by the green line with slope $-1 / 2$.

by Marking Strategy E. This is reported in Table 5.1 for two dimensions (left) and three dimensions (right). We see that the number of additional elements due to large data oscillation is rather small relative to those due to large error indicators, but it is not zero. On the one hand, it confirms that control of data oscillation cannot be omitted in a convergent algorithm. On the other hand, it explains why data oscillation seems to play a minor role for (piecewise) smooth data $f$, and hints at the underlying reasons why most adaptive strategies, although neglecting data oscillation, converge in practice.

As mentioned in section 5.1, we produce in three dimensions the interior node by bisecting a marked tetrahedron six times. This corresponds in two dimensions to four bisections of a marked triangle, which is used here instead of the procedure of Figure 5.1. Although this produces more DOFs than needed, Figures 5.12 and 5.13 demonstrate that the resulting meshes are still quasi-optimal for both two dimensions and three dimensions. Here, the estimators $\eta_{k}$ were scaled by the factor 0.25. For comparison with an optimal mesh, green lines with slope $-1 / d$ are plotted in Figure $5.12(d=2)$ and Figure $5.13(d=3)$; note that these lines have nearly the same slope due to different scaling of the $y$ axis.

Finally, in Figure 5.14 we cut $(0,1)^{3}$ out of the domain $(-1,1)^{3}$ and show the adaptive grid of the three-dimensional simulation on the boundary of the resulting 


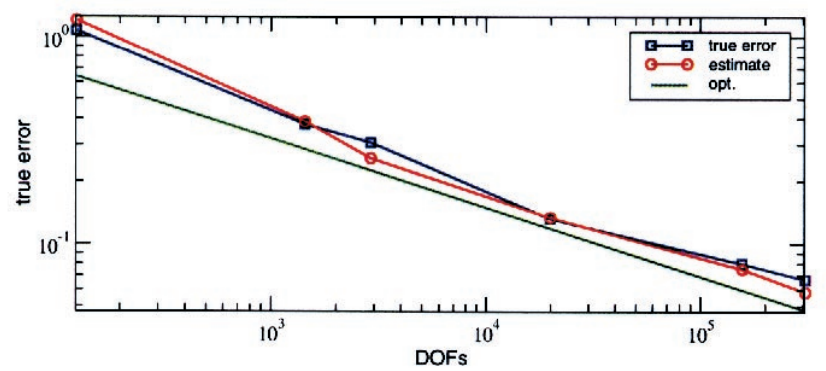

Fig. 5.13 Example 5.4: Quasi-optimality of CONV: estimate and true error in three dimensions. The optimal decay is indicated by the green line with slope $-1 / 3$.
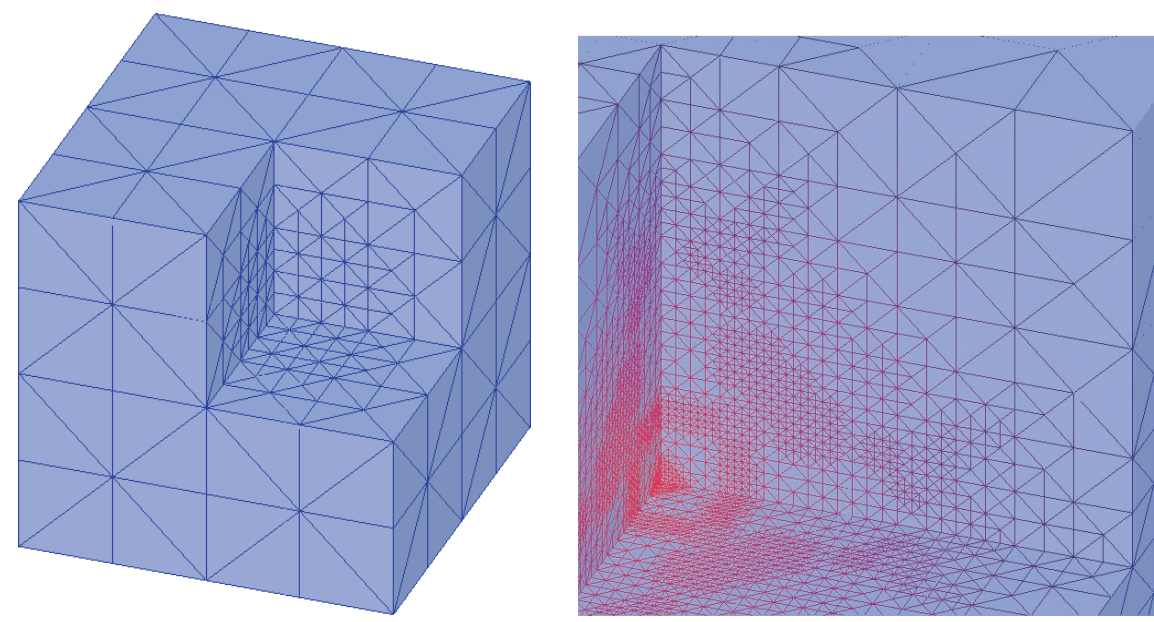

Fig. 5.14 Example 5.4: Adaptive grids of the three-dimensional simulation on $\partial\left((-1,1)^{3} \backslash(0,1)^{3}\right)$ : full grid of the second iteration (left), zoom into the grid of the fourth iteration (right).

domain. In the left picture we show the full grid of the second iteration and in the right one a zoom into the grid of the fourth iteration. For this picture we also used the graphics package GRAPE.

6. Higher Order Elements. For most applications, the solution exhibits enough local regularity for higher order elements to outperform linear elements. In fact, the additional effort of handling more degrees of freedom per element and more dense matrices results in a reasonable gain of precision and overall computational cost. In other words, a drastic reduction of the total number of degrees of freedom, relative to a given error tolerance, is typically observed in practice. This is documented in Figure 6.1, which shows the error decay vs. number of DOFs for polynomial degrees $1 \leq \ell \leq 4$ for a smooth solution of a linear elasticity problem and a solution of Poisson's equation with a point singularity. Especially for the smooth solution the higher order discretization is much superior: the error on the first grid for quartic elements with less than 800 DOFs is already smaller than for the linears on the finest grid with nearly 100,000 DOFs. Even though the solution to Poisson's equation exhibits a point singularity, the higher order discretization pays off in the second 

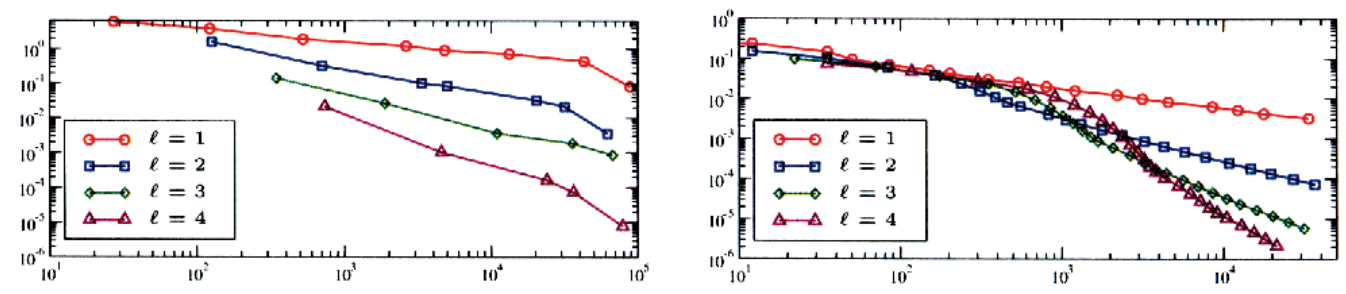

Fig. 6.I Comparison of error decay vs. DOFs for different polynomial degree $\ell$. Left: linear elasticity in three dimensions with a smooth solution; right: Poisson equation in two dimensions with a singular solution.

example shown on the right, as well, especially on finer meshes after resolving the singularity.

The convergence result of section 3 can now be extended to higher order discretizations in the following way: Let $\mathbb{V}_{0}^{H}$ denote now a space of piecewise polynomial functions of degree $\leq \ell$ over the partition $\mathcal{T}_{H}$, which vanish on $\partial \Omega$. The error-residual equation (2.5) remains valid provided the interior residual $f$ is replaced by $R=f+\operatorname{div}\left(A \nabla u_{H}\right)$. As assessed in sections 3 and 4 , a crucial ingredient for convergence is the control of the oscillation $R-R_{T}$ for all $T \in \mathcal{T}_{H}$. Hereafter, $g_{T}$ indicates the $L^{2}$-projection over the space of polynomials of degree $\leq \ell-1$ of a function $g \in L^{2}(T)$. Since $A$ is piecewise constant, then $R-R_{T}=f-f_{T}$. We claim now that the construction of Lemma 4.2, which is instrumental for error reduction, is still feasible provided the interior nodes property (3.1) is verified by any refinement $\mathcal{T}_{h}$ of $\mathcal{T}_{H}$. To see this we simply recall the piecewise linear functions $\varphi_{i}, \varphi_{S}$ of Figure 4.2 and observe that both $f_{T_{i}} \varphi_{i}, J_{S} \varphi_{S} \in \mathbb{V}_{0}^{h} \cap H_{0}^{1}\left(\omega_{S}\right)$ because they are piecewise polynomials of degree $\leq \ell$ over $\mathcal{T}_{h}$. With this at hand, the convergence theory of sections 3 and 4 extends to this case. We refer to [18] for details, simulations, and performance evaluations.

7. Saddle Point Problems. In this section we show convergence of an adaptive Uzawa FEM for the Stokes problem and comment on extensions to other saddle point problems. The chief difficulty in this setting is the lack of a minimization principle and thus the failure of Lemma 4.1, which allows for the quantification of error reduction. Our approach is instead based on [3], which in turn exploits an idea introduced in [7] in the context of wavelet approximations; see also [6]. We refer to [3] for details, extensive computations, and complexity considerations.

7.I. The Stokes Problem. Let $\mathbb{V}:=\left(H_{0}^{1}(\Omega)\right)^{d}$ and $\mathbb{P}:=L_{0}^{2}(\Omega)$ be the subspace of $L^{2}(\Omega)$ of functions with zero mean value. Let $a: \mathbb{V} \times \mathbb{V} \rightarrow \mathbb{R}$ and $b: \mathbb{V} \times \mathbb{P} \rightarrow \mathbb{R}$ be the following continuous bilinear forms:

$$
a(v, w):=\langle\nabla v, \nabla w\rangle, \quad b(v, q):=-\langle q, \operatorname{div} v\rangle \quad \forall v, w \in \mathbb{V}, q \in \mathbb{P} .
$$

Then there exists a unique solution $(u, p) \in \mathbb{V} \times \mathbb{P}$ of the following saddle point problem:

$$
\begin{array}{rlrl}
a(u, v)+b(v, p) & =\langle f, v\rangle & & \forall v \in \mathbb{V}, \\
b(u, q) & =0 & \forall q \in \mathbb{P} ;
\end{array}
$$


see [4, Chapter II]. Let $\mathcal{A}: \mathbb{V} \rightarrow \mathbb{V}^{*}, \mathcal{B}: \mathbb{V} \rightarrow \mathbb{P}^{*}=\mathbb{P}$, and $\mathcal{B}^{*}: \mathbb{P} \rightarrow \mathbb{V}^{*}$, the adjoint of $\mathcal{B}$, be the operators $\mathcal{A}:=-\Delta, \mathcal{B}:=-\operatorname{div}$, and $\mathcal{B}^{*}:=\nabla$. The system (7.2)-(7.3) can be equivalently written in operator form as follows [4]:

$$
\mathcal{A} u+\mathcal{B}^{*} p=f, \quad \mathcal{B} u=0 .
$$

If $S:=\mathcal{B A}^{-1} \mathcal{B}^{*}: \mathbb{P} \rightarrow \mathbb{P}$ denotes the Schur complement operator, which turns out to be positive definite, self-adjoint, and bounded, then $p$ satisfies the equation

$$
S p=\mathcal{B A}^{-1} f .
$$

Since $\alpha:=\|I-\rho S\|<1$ provided the relaxation parameter satisfies $0<\rho<$ $2 /\|S\|_{\mathcal{L}(\mathbb{P}, \mathbb{P})}$, the following Uzawa iteration converges:

$$
p_{k}=p_{k-1}-\rho\left(S p_{k-1}-\mathcal{B A}^{-1} f\right)=(I-\rho S) p_{k-1}+\rho \mathcal{B A}^{-1} f .
$$

We stress that $\rho=1$ is an admissible choice for the Stokes problem, and that this iteration is carried out at the infinite-dimensional level. In weak form it reads

$$
\begin{aligned}
a\left(u_{k}, v\right) & =\langle f, v\rangle-b\left(v, p_{k-1}\right) & & \forall v \in \mathbb{V}, \\
\left\langle p_{k}, q\right\rangle & =\left\langle p_{k-1}, q\right\rangle+\rho b\left(u_{k}, q\right) & & \forall q \in \mathbb{P} .
\end{aligned}
$$

Suppose now that a procedure ELLIPTIC for the operator $\mathcal{A}$, such as described in sections 3 and 4, approximates the solution $u_{k}$ of (7.7) to any desired tolerance $\varepsilon_{k}$ :

$$
\left(\mathcal{T}_{k}, U_{k}\right) \leftarrow \operatorname{ELLIPTIC}\left(\mathcal{T}_{k-1}, P_{k-1}, \varepsilon_{k}, f\right) .
$$

In other words, given $P_{k-1} \in \mathbb{P}_{k-1}$ over the triangulation $\mathcal{T}_{k-1}$, ELLIPTIC constructs a refinement $\mathcal{T}_{k}$ of $\mathcal{T}_{k-1}$ and computes

$$
U_{k} \in \mathbb{V}_{k}: \quad a\left(U_{k}, V\right)=\langle f, V\rangle-b\left(V, P_{k-1}\right) \quad \forall V \in \mathbb{V}_{k},
$$

such that $\left\|U_{k}-u_{k}\right\|_{\mathbb{V}} \leq \varepsilon_{k}$, where $u_{k}$ is the continuous solution of

$$
u_{k} \in \mathbb{V}: \quad a\left(u_{k}, v\right)=\langle f, v\rangle-b\left(v, P_{k-1}\right) \quad \forall v \in \mathbb{V} .
$$

We also assume that a procedure UPDATE for the operator $\mathcal{B}$, namely,

$$
P_{k} \leftarrow \operatorname{UPDATE}\left(\mathcal{T}_{k}, P_{k-1}, U_{k}, \rho\right),
$$

computes a discrete solution of (7.8)

$$
P_{k} \in \mathbb{P}_{k}: \quad\left\langle P_{k}, Q\right\rangle=\left\langle P_{k-1}, Q\right\rangle+\rho b\left(U_{k}, Q\right) \quad \forall Q \in \mathbb{P}_{k} .
$$

If $\Pi_{k}: \mathbb{P} \rightarrow \mathbb{P}_{k}$ denotes the $L^{2}$-projection operator, then (7.13) reads equivalently

$$
P_{k}=P_{k-1}+\rho \Pi_{k} \mathcal{B} U_{k} .
$$

If $j$ is the polynomial degree for velocity, and $\ell$ is that for pressure, it turns out that the pairs of continuous finite element spaces

$$
\mathbb{V}_{k}=\mathcal{P}^{j}\left(\mathcal{T}_{k}\right) \cap \mathbb{V}, \quad \mathbb{P}_{k}=\mathcal{P}^{\ell}\left(\mathcal{T}_{k}\right) \cap \mathbb{P}, \quad \ell=j, j-1 \geq 1,
$$


as well as the discontinuous finite element spaces

$$
\mathbb{V}_{k}=\mathcal{P}^{j}\left(\mathcal{T}_{k}\right) \cap \mathbb{V}, \quad \mathbb{P}_{k}=\mathcal{P}_{d}^{j-1}\left(\mathcal{T}_{k}\right) \cap \mathbb{P}, \quad j \geq 1,
$$

are of interest. Hereafter, $\mathcal{P}_{d}^{j}\left(\mathcal{T}_{k}\right)$ denotes the space of scalar-valued as well as vectorvalued (possibly discontinuous) functions that, restricted to an element $T$, are polynomials of degree $\leq j$ for all $T \in \mathcal{T}_{k}$, and $\mathcal{P}^{j}\left(\mathcal{T}_{k}\right)$ denotes the subspace of continuous functions of $\mathcal{P}_{d}^{j}\left(\mathcal{T}_{k}\right)$. We observe that $\ell=j-1$ in (7.15) corresponds to the popular Taylor-Hood family of finite elements. Any other choice in either (7.15) or (7.16) yields an unstable pair of spaces. These spaces $\left(\mathbb{V}_{k}, \mathbb{P}_{k}\right)$ satisfy [3]:

$$
\left\|\Pi_{k} \mathcal{B} U_{k}-\mathcal{B} U_{k}\right\|_{\mathbb{P}} \leq C_{*} \varepsilon_{k} .
$$

We then have the following convergence result for the Stokes problem, which improves upon the original one in [3].

THEOREM 7.1. Let $\rho>0$ be such that $\alpha=\|I-\rho S\|<1$. Given $\varepsilon_{0}>0$ and $0<\gamma<1$, define $\varepsilon_{k}:=\gamma \varepsilon_{k-1}$. Let the procedures ELLIPTIC and UPDATE satisfy (7.10)(7.14) and (7.17), and let $\alpha_{0}:=\max (\alpha, \gamma)$. Then for every $\beta$ such that $\alpha_{0}<\beta<1$ the iterates $\left(U_{k}, P_{k}\right) \in \mathbb{V}_{k} \times \mathbb{P}_{k}$ satisfy

$$
\left\|p-P_{k}\right\|_{\mathbb{P}} \leq C_{p} \beta^{k}, \quad\left\|u-U_{k}\right\|_{\mathbb{V}} \leq C_{u} \beta^{k},
$$

with

$$
C_{p}:=\left\|p-P_{0}\right\|_{\mathbb{P}}+\frac{\rho\left(1+C_{*}\right) \varepsilon_{0}}{\beta-\alpha_{0}}, \quad C_{u}:=\frac{C_{p}}{\beta}+\varepsilon_{0} .
$$

Proof. In view of (7.10) and (7.14), we see that

$$
\begin{aligned}
P_{k} & =P_{k-1}+\rho \mathcal{B} u_{k}+\rho \mathcal{B}\left(U_{k}-u_{k}\right)+\rho\left(\Pi_{k}-I\right) \mathcal{B} U_{k} \\
& =(I-\rho S) P_{k-1}+\rho \mathcal{B} \mathcal{A}^{-1} f+\rho \mathcal{B}\left(U_{k}-u_{k}\right)+\rho\left(\Pi_{k}-I\right) \mathcal{B} U_{k} .
\end{aligned}
$$

Using (7.5), we readily get the error equation

$$
p-P_{k}=(I-\rho S)\left(p-P_{k-1}\right)-\rho \mathcal{B}\left(U_{k}-u_{k}\right)-\rho\left(\Pi_{k}-I\right) \mathcal{B} U_{k} .
$$

We now use the fact that $\|\mathcal{B} v\|_{\mathbb{P}} \leq\|v\|_{\mathbb{V}}$ for all $v \in \mathbb{V}$, and invoke (7.17), to arrive at

$$
\left\|p-P_{k}\right\|_{\mathbb{P}} \leq \alpha\left\|p-P_{k-1}\right\|_{\mathbb{P}}+\rho\left(1+C_{*}\right) \varepsilon_{k},
$$

whence, by recursion, we end up with

$$
\left\|p-P_{k}\right\|_{\mathbb{P}} \leq \alpha^{k}\left\|p-P_{0}\right\|_{\mathbb{P}}+\rho\left(1+C_{*}\right) \varepsilon_{0} \sum_{j=0}^{k-1} \alpha^{j} \gamma^{k-j} .
$$

Arguing similarly as in Theorem 3.11 , we readily obtain

$$
\left\|p-P_{k}\right\|_{\mathbb{P}} \leq \beta^{k}\left(\left\|p-P_{0}\right\|_{\mathbb{P}}+\frac{\rho\left(1+C_{*}\right) \varepsilon_{0}}{\beta-\alpha_{0}}\right) .
$$

This is the asserted estimate for $\left\|p-P_{k}\right\|_{\mathbb{P}}$. To obtain a similar bound for $\left\|u-U_{k}\right\|_{\mathbb{V}}$, we first observe that

$$
a\left(u-u_{k}, v\right)=b\left(v, P_{k-1}-p\right) \quad \forall v \in \mathbb{V},
$$




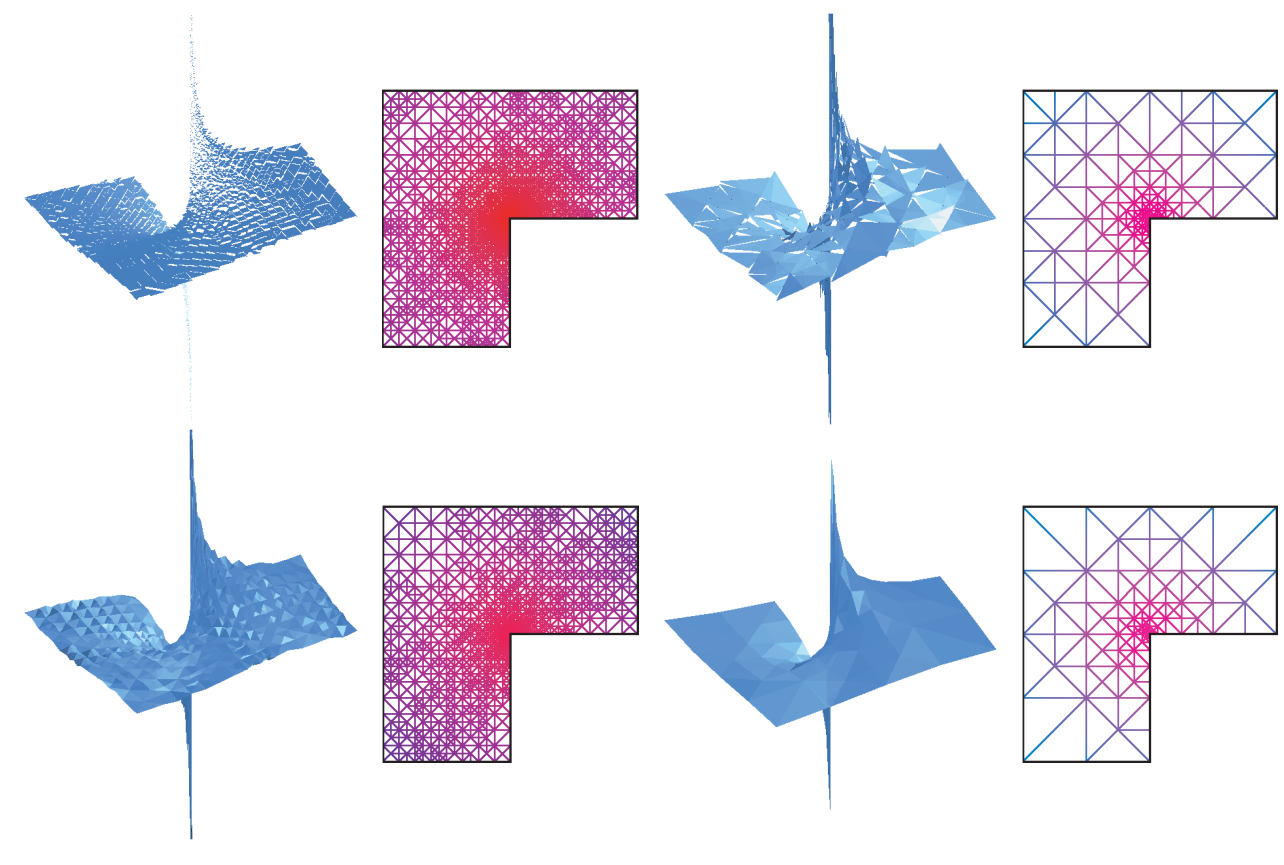

Fig. 7.I Example 7.2: Pressures and meshes for tolerance of 5\% and finite element pairs (resp., outer iteration number $/ D O F s) ; \mathcal{P}^{1} \mathcal{P}_{d}^{0}(50 / 9680), \mathcal{P}^{2}-\mathcal{P}_{d}^{1}$ (35/1940), $\mathcal{P}^{1}{ }_{-} \mathcal{P}^{1}$ (50/4971), $\mathcal{P}^{2}-\mathcal{P}^{1}(50 / 1200)$. The oscillations for unstable elements do not persist under further selective refinement.

whence $\left\|u-u_{k}\right\|_{\mathbb{V}} \leq\left\|p-P_{k-1}\right\|_{\mathbb{P}}$. Since

$$
\left\|u-U_{k}\right\|_{\mathbb{V}} \leq\left\|u-u_{k}\right\|_{\mathbb{V}}+\left\|u_{k}-U_{k}\right\|_{\mathbb{V}} \leq\left\|p-P_{k-1}\right\|_{\mathbb{P}}+\varepsilon_{k},
$$

the remaining estimate for $\left\|u-U_{k}\right\|_{\mathbb{V}}$ follows immediately and finishes the proof.

Remark 7.2. The following statement about the complexity of ELLIPTIC is shown in [3]: if the tolerance reduction factor $\gamma$ of Theorem 7.1 satisfies $\gamma>\alpha$, then the number of iterations of ELLIPTIC is bounded by a constant which depends only on $f$, the initial pressure guess $P_{0}$, the initial triangulation $\mathcal{T}_{0}$, the ratio $\alpha / \gamma$, and the parameters $\theta$ and $\theta_{f}$ of ELLIPTIC, but not on the adaptive counter $k$.

7.2. Example: L-Shaped Domain. We present a simulation on an unit-size Lshaped domain with exact solutions $u \approx r^{1 / 2}$ and $p \approx r^{-1 / 2}$ [3], [21]. We only depict the pressure $P_{k}$ in Figure 7.1, which is the most sensitive variable to instabilities. We point out that the only stable pair is the Taylor-Hood element $\mathcal{P}^{2}-\mathcal{P}^{1}$ (Figure 7.1, right bottom), but that oscillations for unstable pairs do not persist under selective refinement. We refer to [3] for extensive computations showing quasi-optimal meshes for all combinations (7.15)-(7.16) in two and three dimensions.

The computational results corroborate the assertion of Theorem 7.1. We may rephrase this as follows: the addition of the least amount of selective refinement by adaptivity has a stabilizing effect. This is quite different from stabilization techniques based on global refinement for velocity [4], and a surprising outcome of this approach. 
7.3. Mixed Finite Elements of Raviart-Thomas. The theory of section 7.1 extends to abstract saddle point problems (7.2)-(7.3) as long as $a$ and $b$ satisfy

$$
\begin{aligned}
\inf _{v \in \mathbb{V}} \frac{a(v, v)}{\|v\|_{\mathbb{V}}^{2}} \geq c_{a}>0 \quad \text { (ellipticity), } \\
\inf _{q \in \mathbb{P} \backslash\{0\}} \sup _{v \in \mathbb{V} \backslash\{0\}} \frac{b(v, q)}{\|q\|_{\mathbb{P}}\|v\|_{\mathbb{V}}}=\kappa>0 \quad \text { (inf-sup condition). }
\end{aligned}
$$

For the mixed formulation of $(1.2)$, we have that $\mathbb{V}:=H(\operatorname{div}, \Omega), \mathbb{P}:=L^{2}(\Omega)$, and

$$
a(v, w):=\left\langle A^{-1} v, w\right\rangle, \quad b(v, q):=-\langle q, \operatorname{div} v\rangle \quad \forall v, w \in \mathbb{V}, q \in \mathbb{P} .
$$

However, $a$ does not satisfy (7.18). Problem (7.2)-(7.3) now reads

$$
\begin{array}{rlrl}
a(u, v)+b(v, p) & =0 & & \forall v \in \mathbb{V}, \\
b(u, q) & =\langle f, q\rangle & \forall q \in \mathbb{P} .
\end{array}
$$

To enforce ellipticity in $\mathbb{V}$, we augment (7.21) with (7.22) tested against $q=\operatorname{div} v \in \mathbb{P}$ :

$$
a(u, v)+b(u, \operatorname{div} v)+b(v, p)=\langle f, \operatorname{div} v\rangle \quad \forall v \in \mathbb{V} .
$$

The resulting bilinear form

$$
\hat{a}(v, w):=a(v, w)+b(v, \operatorname{div} w)=\langle(I-\nabla \operatorname{div}) v, w\rangle \quad \forall v, w \in \mathbb{V}
$$

is now elliptic in $\mathbb{V}$ and corresponds to the operator $\mathcal{A}:=I-\nabla$ div. Since the RaviartThomas elements satisfy $\operatorname{div} V=\mathbb{P}$, the augmented Lagrangian formulation coincides with the original mixed formulation. We then realize that the theory of sections 3,4 , and 7.1 extends to this case provided a posteriori error estimators can be derived for $\mathcal{A}$, along with an error reduction property. This is indeed the case, and will appear elsewhere.

Acknowledgments. We would like to thank Luis Caffarelli for an inspiring chat and Claudio Verdi for an inspiring Barolo 69.

\section{REFERENCES}

[1] I. BABUŠKA AND A. MilleR, A feedback finite element method with a posteriori error estimations: Part I. The finite element method and some basic properties of the a posteriori error estimator, Comput. Methods Appl. Mech. Engrg., 61 (1987), pp. 1-40.

[2] I. BABUŠKA AND M. Vogelius, Feedback and adaptive finite element solution of onedimensional boundary value problems, Numer. Math., 44 (1984), pp. 75-102.

[3] E. Bänsch, P. Morin, And R.H. Nochetto, An adaptive Uzawa FEM for the Stokes problem: Convergence without the inf-sup condition, SIAM J. Numer. Anal., 40 (2002), pp. 12071229.

[4] F. Brezzi and M. Fortin, Mixed and Hybrid Finite Element Methods, Springer-Verlag, New York, 1991.

[5] A. Cohen, W. Dahmen, And R. DeVore, Adaptive wavelet methods for elliptic operator equations - Convergence rates, Math. Comp., 70 (2001), pp. 27-75.

[6] S. Dahlke, W. Dahmen, And K. Urban, Adaptive Wavelet Methods for Saddle Point Problems-Optimal Convergence Rates, preprint.

[7] S. Dahlke, R. Hochmuth, And K. Urban, Adaptive wavelet methods for saddle point problems, M2AN Math. Model. Numer. Anal., 34 (2000), pp. 1003-1022. 
[8] W. DöRfler, A convergent adaptive algorithm for Poisson's equation, SIAM J. Numer. Anal., 33 (1996), pp. 1106-1124.

[9] W. DöRfler, A robust adaptive strategy for the nonlinear Poisson equation, Computing, 55 (1995), pp. 289-304.

[10] W. Dörfler and R.H. Nochetto, Small data oscillation implies the saturation assumption, Numer. Math., 91 (2002), pp. 1-12.

[11] W. Dörfler And O. Wilderotter, An adaptive finite element method for a linear elliptic equation with variable coefficients, ZAMM Z. Angew. Math. Mech., 80 (2000), pp. 481-491.

[12] GRAPE-GRAphics Programming Environment Manual, Version 5, SFB 256, University of Bonn, Germany, 1995.

[13] R.B. KellogG, On the Poisson equation with intersecting interfaces, Appl. Anal., 4 (1975), pp. 101-129.

[14] I. KossaczkÝ, A recursive approach to local mesh refinement in two and three dimensions, J. Comput. Appl. Math., 55 (1994), pp. 275-288.

[15] W. Mitchell, A comparison of adaptive refinement techniques for elliptic problems, ACM Trans. Math. Software, 15 (1989), pp. 210-227.

[16] P. Morin, R.H. Nochetto, And K.G. Siebert, Data oscillation and convergence of adaptive FEM, SIAM J. Numer. Anal., 38 (2000), pp. 466-488.

[17] P. Morin, R.H. Nochetto, And K.G. Siebert, Local problems on stars: A posteriori error estimators, convergence, and performance, Math. Comp., to appear.

[18] P. Morin, R.H. Nochetto, And K.G. Siebert, Basic Principles for Convergence of Adaptive Higher-Order FEM-Application to Linear Elasticity, in preparation.

[19] A. SChmidt AND K.G. Siebert, ALBERT - Software for scientific computations and applications, Acta Math. Univ. Comenian. (N.S.), 70 (2001), pp. 105-122.

[20] A. Schmidt and K.G. Siebert, alberT: An Adaptive Hierarchical Finite Element Toolbox, Documentation, Preprint 06/2000, Universität Freiburg, available online from http://www.mathematik.uni-freiburg.de/IAM/ALBERT.

[21] R. Verfürth, A Review of A Posteriori Error Estimation and Adaptive Mesh-Refinement Techniques, Wiley-Teubner, Chichester, UK, 1996. 